Document downloaded from:

http://hdl.handle.net/10251/104021

This paper must be cited as:

Torregrosa, AJ.; Serrano, J.; Piqueras, P.; Garcia Afonso, O. (2017). Experimental and computational approach to the transient behaviour of wall-flow diesel particulate filters. Energy. 119:887-900. doi:10.1016/j.energy.2016.11.051

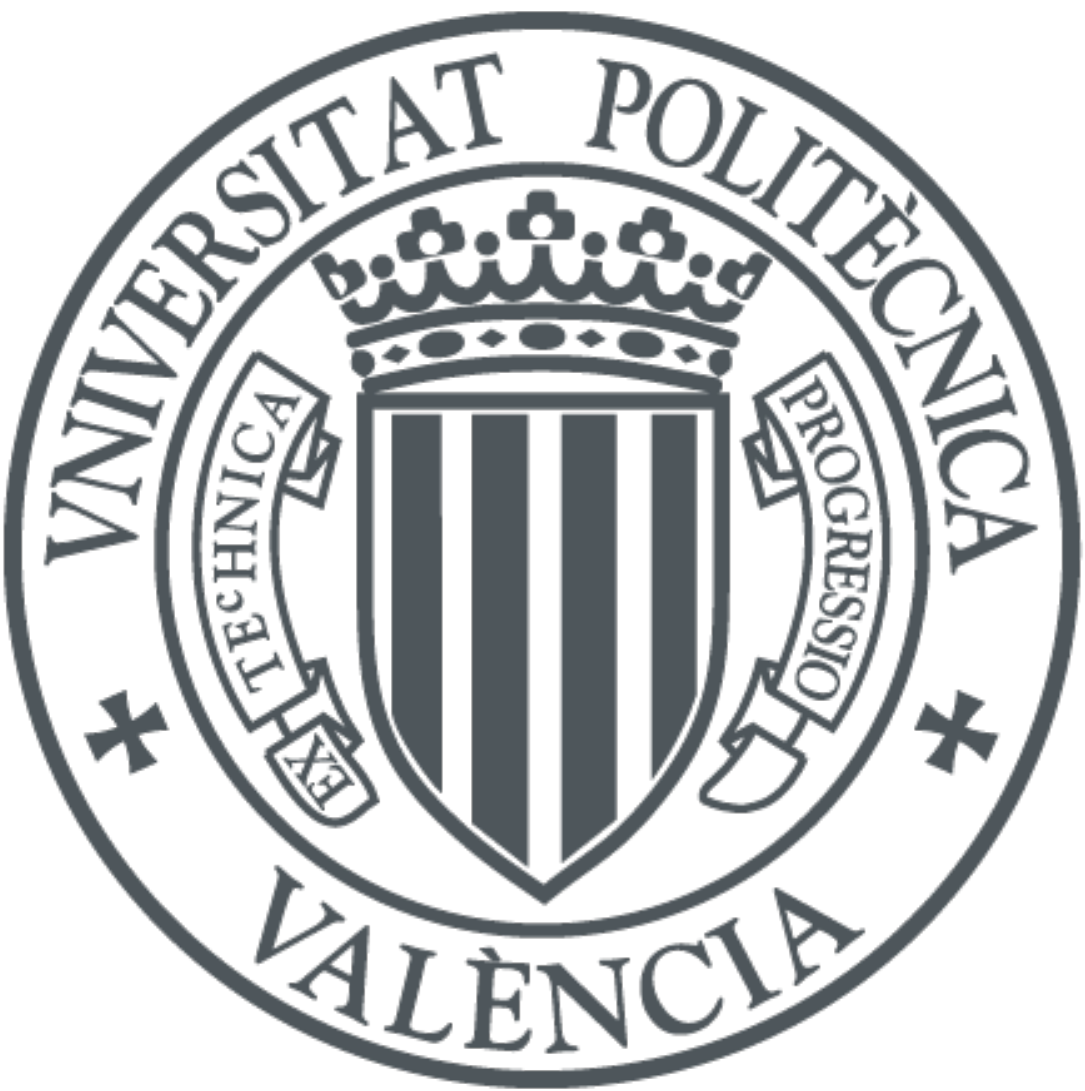

The final publication is available at

http://dx.doi.org/10.1016/j.energy.2016.11.051

Copyright Elsevier

Additional Information 


\title{
Experimental and computational approach to the transient behaviour of wall-flow diesel particulate filters
}

\author{
Antonio José Torregrosa, José Ramón Serrano, Pedro Piqueras*, Óscar García-Afonso \\ CMT-Motores Térmicos, Universitat Politècnica de València, Camino de Vera s/n, 46022 Valencia, Spain.
}

\begin{abstract}
The implementation of tight vehicle emission standards has forced manufactures to use aftertreatment systems extensively. In addition to pollutant emissions abatement, these devices have a noticeable impact on the wave pattern. This fact affects the muffler design criteria. All monolithic aftertreatment devices produces a damping effect because of the honeycomb structure and the narrow channels. However, this response is more marked in wall-flow diesel particulate filters (DPF) because of the alternatively plugged ends and the dissipative properties of the porous substrate.

The main goal of this paper is to assess the transient fluid-dynamic behaviour of wall-flow DPFs using experimental and modelling techniques. The experimental data were gathered in clean and loaded conditions. The DPF was subjected to a variety of pressure excitations to characterise its transient behaviour in the time and frequency domains. Afterwards, the DPF response was evaluated under engine-like operating conditions in an unsteady flow gas stand. Once the main characteristics of the response were known, a non-linear gas-dynamics model was proposed for analysis and prediction. The model accounts for space and time gradients, combining the thermo-and fluid-dynamic solution with a model based on a packed bed of spherical particles that defines the meso-structure of the loaded substrate.
\end{abstract}

Keywords: Diesel engines; aftertreatment; diesel particulate filter; fluid-dynamics; acoustics

\section{Introduction}

As a result of the restrictions imposed by regulations on particulate matter emissions from compression ignition engines, diesel particulate filters (DPFs) have become an indispensable device in exhaust lines [1]. Similarly, current and incoming standards on spark ignition engines combined with developments associated with turbocharged and direct injection gasoline engines [2] are also demanding the use of gasoline particulate filters (GPF) as the only system proven to fulfill limits on emitted particle numbers [3].

The development of particulate filters is constrained by the need of high filtration efficiency, so that standards in mass and number of emitted particles are fulfilled, together with a reasonable pressure drop. A good trade-off between these two aspects must be complemented with suitable characteristics of the substrate making possible safe

${ }^{*}$ P. Piqueras. CMT-Motores Térmicos, Universitat Politècnica de València, Camino de Vera s/n, 46022 Valencia, Spain. Phone: +34 963877650 Fax: +34 963877659 e-mail: pedpicab@mot.upv.es 
and reliable regeneration as well as providing good thermal and mechanical responses [4]. These characteristics define the filter capability to abate particulate matter emissions, its influence on engine performance and the life cycle and maintenance requirements of the system.

The complexity of the concurrent phenomena has given rise to the study of alternatives, comprising the consideration of different substrate materials [5] and even flow path-lines [6]. Among all of them, cordierite and SiC wall-flow monoliths have shown the best balance between all requirements [7]. Therefore, manufacturers have massively installed wall-flow DPFs in both heavy-and light-duty compression ignition engines since their use became commonplace at the beginning of the present century [8].

Wall-flow DPFs are monolithic structures with alternatively plugged axial parallel channels at each end. The exhaust gas goes into the inlet channels and finds the closed end so that it is forced to flow across the porous wall of the ceramic substrate. During this process, particles are firstly collected inside the porous wall in a regime known as deep bed filtration. When the porous wall gets saturated, the soot is then deposited on its surface forming the particulate layer and reaching filtration efficiency above 95\% even for ultra-fine particles [9]. However, the geometrical characteristics of this solution lead to a non-negligible pressure drop that increases the exhaust back-pressure, specially as the DPF gets loaded. This effect directly implies a fuel penalty [10] to which is added the subsequent regeneration. In fact, the most effective way to promote soot oxidation appears to be the use of active strategies such as late fuel injection [11], $\mathrm{NO}_{2}$ assisted passive regeneration [12] or the use of fuel-borne catalysts [13].

Strategies aiming to reduce the wall-flow DPF effects on engine fuel economy are mostly based on pressure drop control. Promising solutions, such as the optimization of DPF sizing [14] or the use of inhomogeneous substrates [15], pre-turbo DPF location [16] and pre-DPF water injection [17] have been proposed. Most of these strategies have also very positive effects on passive regeneration capability [18] and provide higher flexibility than active regeneration control strategies based exclusively on pressure drop [17]. The benefits brought by these techniques cover also the effects of ash [19] and their ability to be combined with particular solutions aiming at increasing ash storage capacity, such as asymmetrical cell design [20].

Although the main purpose of the wall-flow DPF is the reduction of particulate matter emissions, its presence affects the unsteady wave dynamics in the exhaust system [21]. The flow path, the canning and the absorbent ceramic material composing the wall-flow monolith introduce a dissipative effect upstream of the muffler [22]. This modifies the boundary conditions for muffler design, usually leading to a substantial volume reduction [23] or even to its removal in cases of severe packaging restrictions [24], with the subsequent repercussion on exhaust backpressure. The use of computational models able to predict the DPF behaviour under different soot loading and unsteady flow conditions are an advantage to reduce the time and cost of the development process.

Fist attempts to model unsteady flow in catalytic honeycomb structures were restricted to the linear regime and date back to the work of Glav et al. [25]. The first work exclusively devoted to wall-flow DPFs was presented by Allam and Åbom [26], who proposed a linear 1D model. The main limitation of this first effort was the lack of wall boundary layers, that were included in later works [21]. Finally, they developed a new version of the model including 
a correct description of the visco-thermal boundary layers for square cross-section channels [27]. The model proposed by Allam and Åbom is the basis for the definition of the transfer matrix in other linear models such as the 3D-FEM models proposed by Hua et al. [22] to improve the prediction of the DPF response at very high frequencies. FEM modelling has been also explored by Gao et al. [28] with further discussion on the correction of the viscosity. Recently Hua et al. have explored the validity of the plane wave assumption in large monoliths by means of BEM and FEM techniques. An important influence of the inlet and outlet characteristics on the design of the muffler system has been found [29].

The purpose of this paper is to assess the behaviour of wall-flow DPFs in unsteady flow by combining experimental and modelling approaches. The experimental campaign was the basis for the subsequent computational study and covered clean and soot-loaded DPF conditions. For every soot loading condition, the DPF was firstly subjected to tests in an impulse test rig, i.e. to unsteady pressure excitations at room temperature. The unsteady behaviour under engine-like operating conditions was evaluated in a gas stand equipped with a rotary valve providing a periodic excitation superimposed to a mean flow. The use of this facility allowed controlling the flow characteristics, such as the gas temperature, while keeping constant the soot loading. Finally, for the proper interpretation of the experimental results a non-linear gas dynamic model of the DPF was developed. The proposed model solves the 1D compressible unsteady non-homentropic governing equations [30] in the monolith channels as well as the inertial pressure drop in the inlet and outlet canning volumes. Temperature gradients in the axial and radial directions are described by means of a heat transfer sub-model based on nodal discretization of the monolith porous medium [31]. The change of the meso-structural properties of the porous medium, for both the porous substrate and the particulate layer, are computed as a function of the soot loading [32] assuming an equivalent packed bed of spherical particles [32]. These features of the model provide an accurate description in the time and frequency domains of the observed effects in terms of pressure drop and pressure wave transmission and reflection.

\section{Experimental facilities and test campaign}

The unsteady behaviour of the DPF was explored considering two basic test facilities: experiments without mean flow in an impulse test rig and experiments with mean flow in a flow test rig able to provide pulsating flow with temperature control. The main characteristics of the tested DPF are summarised in Table 1. All tests were performed with fresh air. Although the use of air instead of simulated exhaust gas has effects on the fluid thermal properties and hence on the speed of sound, the use of air is preferable. The most important reason is the need to avoid the variation of the soot loading during the tests. Use of air allows keeping constant soot loading in every kind of test and provides flexibility and repeatability to extend the test campaign. Additionally, it use defines a clear baseline for thermal properties assessment since the specific heat is only dependent on temperature but not on the fuel-air equivalence ratio, which should be considered as additional parameter in the case of exhaust gas use. 


\subsection{Impulse test rig}

Experiments without mean flow were performed in an impulse test rig able to control the amplitude and the duration of an isolated pressure excitation travelling along the piping installation and the DPF. This kind of test allows evaluating the reflection and transmission characteristics of the system avoiding any influence of reflections from the pipe ends of the test rig. Payri et al. [33] presented the experimental method applied in the impulse test rig for the measurement of the frequency response to weakly nonlinear transient excitations. The validation of the method was performed against one-dimensional unsteady nonlinear flow calculations of well-known simple filter geometries. In some cases, only quantitative deviations from the expected linear behaviour were observed, due to the extra amount of dissipation associated with nonlinear flow. In other cases, however, qualitative differences may appear, with changes in the detail of the results which, in principle, can be explained by attending to the possibility of nonlinear energy exchange between harmonics.

Figure 1 shows schematically the setup of the test rig [33]. The incident pulse is generated at room temperature by means of a high-speed electrovalve that controls the discharge from a pressurised air tank. The electrovalve is connected to a long duct through which the incident pulse propagates. The length of the propagation duct up to the DPF is selected to ensure that the pressure transducer 1 measures only the generated pressure pulse once it is developed, and without any overlap with the pulse reflected by the DPF. Piezoelectric pressure sensors Kistler 7001 are used in impulse gas stand because of their high sensitivity, which makes them especially suited for dynamic pressure measurements. The characteristics of this kind of sensor, which is coupled to a charge amplifier Kistler 5011B, are summarised in Table 2.

When the incident pulse arrives at the DPF, a reflected wave is generated. It travels back to the electrovalve, which is kept closed. The superposition of the incident and reflected pulses is registered by pressure transducer 2. Finaly, pressure transducer 3 registers the pressure pulse transmitted downstream of the DPF, which is not affected by any reflection from the discharge end because of the long length of the outlet duct.

Due to the fact that transducer 2 registers the superposition $\left(p_{c}\right)$ of the incident and reflected pulses, an additional test with a straight duct portion instead of the DPF is performed in order to identify the incident wave. Firstly the straight duct is tested being subjected to several incident pressure pulses with similar (within a prescribed uncertainty) duration and amplitude. Afterwards the straight duct portion is replaced by the DPF to be tested and again several measurements are performed with pulses similar to those used with the duct portion. Then, the measurements of pressure transducer 1 in the two tests are compared to find the most coincident pair of duct and DPF tests by means of a statistical procedure. From this selection of tests, the incident pressure pulse $\left(p_{i}\right)$ at the DPF inlet is defined as the pressure pulse measured by pressure transducer 2 in the case of the straight duct portion. Then, the reflected pressure pulse $\left(p_{r}\right)$ by the DPF is calculated from the measurements of pressure transducer 2 in both tests according to Eq. 1,

$$
\left(\frac{p_{r}}{p_{0}}\right)^{\frac{\gamma-1}{2 \gamma}}=\left(\frac{p_{c}}{p_{0}}\right)^{\frac{\gamma-1}{2 \gamma}}-\left(\frac{p_{i}}{p_{0}}\right)^{\frac{\gamma-1}{2 \gamma}}+1
$$


where $p_{0}$ is the pressure of the unperturbed medium. Table 3 shows the test matrix defining the amplitude and duration of the pressure excitations. Amplitude covers from 60 to $200 \mathrm{mbar}$ and duration from 8 to $20 \mathrm{~ms}$, which are representative of the variety of pressure pulses arriving at the DPF under regular operation in post-and pre-turbo placement. The study included two extreme scenarios of soot loading conditions to completely capture the change in unsteady response: clean DPF and high soot loading $(60 \mathrm{~g}-22 \mathrm{~g} / \mathrm{l})$.

\subsection{Unsteady flow gas stand}

Tests with unsteady periodic flow completed the experimental analysis accounting for the influence of the DPF soot loading and pressure pulses. The schematic setup of the flow gas stand is shown in Figure 2(a). Air is pumped from ambient conditions into the DPF by means of a screw compressor whose speed determines the amount of flow. Downstream of the screw compressor, the air flow is heated up by a set of electrical heaters arranged in parallel. Next the flow is driven to a rotary valve with a by-pass system, as sketched in Figure 2(a), in order to generate the pressure fluctuations. The amplitude and the frequency of these fluctuations are controlled by the rotary valve speed and the by-pass valve opening. This allows generating flow conditions close to those found in an engine exhaust line.

The instrumentation of the gas stand comprises sensors for the control and characterisation of the device operating conditions. Flow temperature was measured by means of four thermocouples located in cross-sections $0.9 \mathrm{~m}$ away from the inlet and outlet of the DPF. The air mass flow was measured with a hot-film anemometer placed downstream of a heat exchanger that cools down the flow close to the gas stand tailpipe. The measuring range is $0-720 \mathrm{~kg} / \mathrm{h}$ with a maximum error of $\pm 1 \%$ FSO. Finally, the instantaneous absolute pressure was registered by means of Kistler 4045A5 piezoresistive sensors with original cooler adapter installed $0.95 \mathrm{~m}$ upstream and downstream of the DPF. Their characteristics are detailed in Table 2.

A beamforming technique [34] was applied upstream and downstream of the DPF. The objective of this technique is to estimate pressure fluctuations by means of the decomposition of the flow into its forwards and backwards wave components, as shown in Figure 2(b). It allows the evaluation of the sound pressure level reflected and transmitted by the device. In particular, beamforming is a spatial processing technique in which the signal waveform arriving from a given direction is identified by means of an array of sensors. Instantaneous pressure measurements are required at three different equally-spaced axial positions along the pipe. In this work, Kistler 7061B cooled piezoelectric transducers coupled to Kistler 5011B charge amplifiers were used. Their main characteristic is the high sensitivity, which enables the detection of pressure fluctuations lower than $200 \mathrm{~Pa}$ even at high temperature. The beamforming procedure used assumes that wave propagation between sensors is linear, which is acceptable if the array aperture (total length) is small compared with the wavelength. The analysis of the measurement error influence of this technique performed by Piero et al. [34] revealed that a maximum relative error in the sound pressure level of $\pm 0.7 \%$ can be assumed for each transducer

The DPF was tested in clean and soot-loaded conditions with different mass flows and temperatures. The device was excited with a variety of pressure fluctuations covering a wide range of frequencies and amplitudes representative 
of the operating conditions that can be found in both post-and pre-turbo DPF placements. Table 4 summarises the whole test matrix of the study. The minimum pulse amplitude used was 0.12 bar in all the cases, and the maximum depends on the mass flow because of the DPF back-pressure and its combination with the regulation capability of the facility. Consequently, it varies between 0.25 bar and 0.4 bar. The sensitivity of the acoustic response to temperature change was assessed by repeating the test in clean and soot loading conditions at $40{ }^{\circ} \mathrm{C}$ and $250{ }^{\circ} \mathrm{C}$. Maximum temperature was limited to avoid the regeneration in presence of $\mathrm{O}_{2}$, which leads to soot oxidation over $300{ }^{\circ} \mathrm{C}$ with rapid increasing reaction rate as temperature does [35]. The interest for temperature is its influence on the speed of sound, i.e. on the perturbation propagation velocity, and on the pressure drop. This last happens in two different ways. On the one hand, the temperature increase leads to lower density in any particular operating condition. Consequently, an increase of gas velocity takes place, what involves the increase of the inertial pressure drop since it is linearly dependent on density but squarely on velocity. On the other hand, the temperature increase produces the increase of the porous medium permeability because of the slip-flow effect. This phenomenon has been proved to be relevant on pressure drop in several research works [36-38]. The consequence is the decrease of the porous media pressure drop in contrast to inertial contributions.

The pulse frequency was selected based on the achievement of representative equivalent engine speeds. Table 5 details the equivalence between the frequency of the fluctuation generated by the rotary valve and the rotating speed of a four-stroke engine with different numbers of cylinders.

\section{Analysis of the experimental results}

The variations in permeability and geometry of the inlet channels that the DPF monolith cyclically undergoes because of the loading and regeneration processes give rise to a significant change in its dynamic response. Figure 3 represents the experimental dynamic response of the DPF in the time domain obtained in the impulse test rig. Clean and soot loaded DPF operating conditions are compared. Every row of plots represents the incident, reflected and transmitted waves corresponding to different excitations.

Given a soot loading condition, results in Figure 3 show that the trend in the reflected and transmitted pressure pulse is approximately scaled with the change of the amplitude and duration of the excitation, whereas significant changes appear when the soot loading is modified. In the case of the clean DPF, two distinct wave patterns are observed in the reflected pulse. In the first instance, a sudden pressure reduction of short duration takes place caused by the cross-section area increase defining the inlet cone. Secondly, there is a compression pulse related to the crosssection area reduction at the frontal face and to the inlet channels closed end. Therefore, one would expected that the importance of this second reflection is larger for the DPF with soot loading $(22 \mathrm{~g} / 1)$. In fact, for this specific case, which represents a high soot loading level, it can be seen in Figure 3 that the amplitude of the reflected pulse has been amplified up to values reaching $45-60 \%$ of the incident pulse amplitude, percentage that increases with the incident amplitude. This indicates that the corresponding increase in the instantaneous velocity gives rise to a higher influence 
of the reflection caused by the soot accumulation at the rear end of the inlet channels. This effect is even more evident because of the decrease of the effective permeability and the increase of the particulate layer thickness. These new conditions also cause the reflected compression pulse to arrive at the measurement station earlier than for the clean DPF, virtually masking the initial rarefaction phase.

These changes in the monolith and porous medium properties also affect the characteristics of the transmitted pulse. Under clean DPF conditions, a significant deformation of the initial pressure rise is observed indicating the influence of both reactive and dissipative effects. Additionally, the amplitude is reduced by around $30 \%$ for all the excitation amplitudes. This trend admits a first quasi-steady interpretation in terms of Darcy's law, since filtration velocity, which increases with amplitude, is proportional to the pressure drop. Such interpretation is also consistent with the behaviour observed in the reflected wave. This fact suggests an accumulation of the flow at the rear end of the inlet channels, leading to an increase in the filtration velocity in this region and hence to a higher pressure drop. When the DPF gets loaded the transmitted pulse is similar in shape although with the expected increase in attenuation due to the permeability reduction and to the presence of the particulate layer.

The corresponding results in the frequency domain are shown in Figure 4. In addition to the reflected and transmitted sound pressure level (SPL) spectra, the transmission loss TL (i.e. the ratio between the incident and the transmitted power) has been plotted. In general terms, no substantial influence of the excitation amplitude is observed on the shape of the reflected and transmitted SPL as well as the TL. In the case of the clean DPF, also the TL values are essentially independent of the excitation amplitude, and the reflected and transmitted SPL roughly scale with it. However, an increase in the TL with the amplitude is observed when the DPF is loaded. This behaviour indicates the presence of non-linearities likely to be related with the correspondingly increasing local values of the filtration velocity.

Considering the effect of filter loading on the reflected SPL, an increase is found for frequencies below $50 \mathrm{~Hz}$ and above $300 \mathrm{~Hz}$. For the two lower amplitude cases, the reactive feature appearing in the clean DPF around $500 \mathrm{~Hz}$ disappears and the loaded spectrum remains flat up to $1000 \mathrm{~Hz}$. In the case of the highest amplitude, this reactive trough is shifted to $700 \mathrm{~Hz}$.

By contrast to the reflection behaviour, the transmitted SPL shows a clear decrease (with the corresponding increase in the TL) when the DPF gets loaded due to the dissipative effect indicated by the increase observed in pressure drop. Broadband attenuation is obtained due to the resistive characteristics of the substrate, as clearly indicated by the transmission loss pattern; however, it is interesting to note how the reactive nature of the canning is apparent in the shape of the transmitted SPL and the transmission loss. Besides the appearance of reactive features at and above $700 \mathrm{~Hz}$, the peak in transmission loss that appears at $300 \mathrm{~Hz}$ gets more much marked under soot loading conditions than with the clean DPF. This fact might be related to the change in the open frontal area at the monolith inlet because of the cross-section decrease caused in the inlet channels by the presence of the soot layer.

The wave decomposition at the inlet and outlet of the DPF with periodic flow is shown in Figure 5. Incident and reflected waves at the DPF inlet and the transmitted wave at the DPF outlet for clean and soot-loaded DPF conditions are compared at two operating points. Left column results correspond to a mean flow of $250 \mathrm{~kg} / \mathrm{h}$ at hot 
flow $\left(250^{\circ} \mathrm{C}\right)$, with pulse amplitude and frequency of 0.25 bar and $66.6 \mathrm{~Hz}$ respectively. The right column shows a case corresponding to $375 \mathrm{~kg} / \mathrm{h}$ and cold flow $\left(40^{\circ} \mathrm{C}\right)$ with the same frequency and a pulse amplitude of 0.12 bar, which is to the lowest value tested. In contrast to impulse tests, the incident pressure does not coincide in these cases between clean and loaded DPF conditions. This is due to the fact that the generated pressure, which is monitored to set frequency and amplitude, is composed of the incident and the reflected wave. Therefore, the change in the reflection from clean to loaded conditions gives rise to a different incident pressure. Additionally, since the outlet pressure of the installation is kept the same, the transmitted wave is almost identical in clean and loaded conditions so that the increase of pressure drop due to soot loading reverts on a mean pressure increase affecting both the incident and the reflected wave.

The frequency domain results are shown in Figures 6-8. The tests represented in Figures 6 correspond to the same operating points as in Figure 5. It is observed that the differences in SPL between clean and loaded conditions are relatively small for all the harmonics both in the reflected and the transmitted wave. Only a slight SPL reduction is found when the DPF is loaded.

In order to verify this trend, a summary of the reflected and transmitted SPL is given in Figures 7 and 8 respectively. The results comprise the second and fourth harmonics in plot sets (a) and (b) for all tested operating points, i.e. swept in amplitude and frequency of the pulses, soot loading, air mass flow and temperature. The left column represents the results corresponding to clean DPF whereas the right column refers to soot loaded conditions; the first row in every plot set corresponds to cold gas temperature and the second row to high gas temperature.

The results depicted in Figure 7 indicate that the magnitude of the reflected SPL increases with the pulse amplitude, i.e. as the gradient of instantaneous velocity grows. However, mass flow has not any evident influence as the change in mean velocity is not affecting the reflected SPL. The influence of the gas temperature is also negligible, especially in the case of the fourth harmonic. Results corresponding to the second harmonic show a slight sensitivity to temperature at the lowest frequency $(33 \mathrm{~Hz})$, at which the SPL decreases. With respect to the influence of the pulse frequency there is not a clear trend. The general tendency in the case of clean conditions and the second harmonic is a decrease of the reflected SPL as the frequency increases. However, in the fourth harmonic the minimum SPL is clearly located at $66 \mathrm{~Hz}$ in all the cases.

It appears anyway that the measured response under soot loading conditions is spurious. Only a reduction in SPL with respect to the clean DPF at the same operating conditions can be confirmed. All mass flows and pulse amplitudes show the same behaviour at given gas temperature and harmonic. However, comparison between different order harmonics reveals that trends are also different to those obtained in clean conditions. In addition, the change of the gas temperature for any harmonic gives rise to additional variations affecting the correlation with pulse frequency. Since the population of operating conditions at high temperature under soot loading conditions is small, the apparent trend indicating an increase of the reflected SPL with frequency cannot be completely confirmed.

With respect to the transmitted SPL, the experimental results represented in Figure 8 evidence that the influence of the pulse amplitude is the same as that observed in the reflected SPL. The increase in the amplitude gives rise to 
an increase of the SPL for all harmonics, independently of the soot loading and gas temperature. Under clean DPF conditions, the maximum SPL of the second harmonic is located at $66 \mathrm{~Hz}$ at low temperature. This feature is again slightly modified by the increase in temperature, which shifts the maximum SPL towards lower frequencies. This same tendency is observed in the fourth harmonic for low and high gas temperature. In contrast to the reflected SPL, the soot loading does not cause any change in the trend with respect to clean DPF conditions, but only a decrease of the transmitted SPL magnitude.

\section{Wall-flow diesel particulate filter model}

A non-linear DPF model integrated into the gas dynamic code OpenWAM ${ }^{\mathrm{TM}}$ [39], which is an open source gas dynamic software [40], has been used to support the analysis of results. The model includes the whole DPF canning, i.e. inlet and outlet volumes and the monolith to properly account for the different contributions to pressure pulse propagation and to pressure drop. The volumes are solved as $0 \mathrm{D}$ elements by means of a filling and emptying model and are coupled to the 1D elements, i.e. exhaust piping and monolith channels. The coupling is done by boundary conditions that solve the inertial pressure drop taking place due to flow expansion or diffusion. These pressure drop contributions are relevant in real DPF applications [30]. This makes its modelling required to take into account nonlinear effects usually neglected when calculation is only focused on the characterisation of the DPF monolith [21]. In the work of Allam and Åbom [21], the plane wave (1D) solution is obtained along the monolith channels with a linear approach. Nevertheless, the proposed model accounts for gradients in the flow properties. Despite the DPF monolith is placed in an expansion chamber, time derivatives and space gradients along the DPF are not negligible for high wave amplitudes and medium to high volumetric flow rates [30]. In these conditions, the filtration velocity gradient becomes high at the rear end of the inlet channels because of the flow accumulation, as shown in Figure 10. Therefore, the prediction of the distribution of flow properties becomes essential for an accurate description of wave reflection and transmission.

According to this behaviour, the model solves the governing equations for non-homentropic one-dimensional unsteady compressible flow including the source terms to account for the flow exiting the inlet channels across the porous wall and entering into the outlet channels [30]:

- Mass conservation

$$
\frac{\partial\left(\rho_{j} F_{j}\right)}{\partial t}+\frac{\partial\left(\rho_{j} u_{j} F_{j}\right)}{\partial x}=(-1)^{j} 4\left(\alpha-2 w_{p l} j\right) \rho_{j} u_{w_{j}}
$$

- Momentum conservation

$$
\frac{\partial\left(\rho_{j} u_{j} F_{j}\right)}{\partial t}+\frac{\partial\left(\rho_{j} u_{j}^{2} F_{j}+p_{j} F_{j}\right)}{\partial x}-p_{j} \frac{d F_{j}}{d x}=-F_{w} \mu_{j} u_{j}
$$

- Energy conservation 


$$
\begin{aligned}
& \frac{\partial\left(e_{0 j} \rho_{j} F_{j}\right)}{\partial t}+\frac{\partial\left(h_{0 j} \rho_{j} u_{j} F_{j}\right)}{\partial x}= \\
& q_{j} \rho_{j} F_{j}+(-1)^{j} 4\left(\alpha-2 w_{p l} j\right) h_{0 w} \rho_{j} u_{w_{j}}
\end{aligned}
$$

In equations (2)-(4), subscript $j$ refers to the type of monolith channel and accounts for the existence of a particulate layer, taking values 0 and 1 in the outlet and the inlet channels, respectively.

The solution of the governing equations in a pair of inlet and outlet channels is coupled through the flow across the porous medium. Therefore, the system of governing equations is closed with the ideal gas state equation applied to the inlet and outlet channels and with the equation governing the pressure drop across the porous medium, which is based in the application of the Darcy's law to the porous wall and the particulate layer [30]. Therefore, assuming incompressible unsteady flow across the porous wall, the filtration velocity in the inlet channel is obtained as

$$
u_{w_{1}}=\frac{p_{1}-p_{0}}{\frac{\mu_{1} w_{w}}{k_{w}} \frac{\rho_{1}\left(\alpha-2 w_{p l}\right)}{\rho_{0} \alpha}+\frac{\mu_{1}\left(\alpha-2 w_{p l}\right)}{2 k_{p l}} \ln \left(\frac{\alpha}{\alpha-2 w_{p l}}\right)},
$$

and applying the continuity equation across the porous medium, in the outlet channel one gets:

$$
u_{w_{0}}=\frac{u_{w_{1}} \rho_{1}\left(\alpha-2 w_{p l}\right)}{\rho_{0} \alpha}
$$

The numerical solution of the set of governing equations in the internal nodes of the channels is performed by means of shock capturing methods. In this work the two-step Lax\&Wendroff method [41] adapted to porous wall channels [30] is used. Additionally, the obtained solution is coupled with a flux-corrected transport technique (FCT) in order to avoid spurious oscillations around discontinuities [42]. A mesh size of $25 \mathrm{~mm}$ was imposed in all experiments to discretise the channels in the axial direction. The solution of the flow along the channels is completed with the use of the Method of Characteristics (MoC) also adapted to solve the boundary conditions in porous wall channels [43]. Therefore, the derived solution of the Riemann variables and the entropy level includes the variation along the spacetime plane due to cross-section area changes, friction and heat transfer as traditionally stated, but also takes into account the influence of the flow leaving or entering to the channels through the porous walls. Two types of boundary conditions are considered. On the one hand, the connections of the monolith channels to the inlet and outlet volumes of the DPF canning. On the other hand, the closed ends due to the plugged end in the inlet and outlet channels.

The solution of a single pair of inlet and outlet channels has been proved to be representative of the pressure drop behaviour of the whole monolith $[44,45]$. This approach is considered to model impulse test rig experiments and tests at low temperature carried out in the unsteady flow test rig. In addition, the model enables the radial discretization of the monolith into concentric channel beams. This feature allows taking into account temperature variations in the radial direction [31]. When this radial discretization is applied only one pair of inlet and outlet channels is solved per each channel beam, so that all channel pairs in the same beam are assumed to behave in the same way. This second approach was the one applied to model high temperature experiments carried out in the unsteady flow test rig. The 
radial mesh consisted of 10 concentric channels beams distributed homogeneously. Figure 9 shows schematically the way in which the DPF monolith is discretised in the axial and radial directions.

The setup of the model was completed with the definition of the boundary conditions defining every experiment. These boundary conditions are different depending on the test being modelled. In the case of tests performed in the impulse test rig, the experimental incident pressure was imposed at the same distance of the DPF inlet at which it was measured by pressure transducer 2 (Figure 1). A duct solved as a 1D element was included to link this boundary condition with the DPF inlet volume. At the DPF outlet another duct was placed to connect up the DPF outlet volume and a boundary condition placed at the same distance as pressure transducer 3 (Figure 1). This boundary condition is an anechoic end according to the test rig design features. In this way, the DPF transmitted pressure can be computed appropriately.

Similarly, the boundary condition at the inlet of the DPF for unsteady flow tests modelling was defined by the instantaneous pressure. Mean value of the temperature provided by the thermocouple was also imposed. The DPF outlet was linked with a duct of the same length that the one in the gas stand. In this case, an open end to room conditions defined the end boundary condition.

\subsection{Porous medium properties}

The modelling of the porous medium properties is a key factor because of their effects on the filtration velocity distribution along the channels. As shown in Eqs. (5) and (6), the filtration velocity is a function of the permeability of the porous substrate $\left(k_{w}\right)$ and the particulate layer $\left(k_{p l}\right)$. In turn, the permeability depends on meso-structure properties such as the porosity and the mean pore diameter [46] as well as on the slip-flow effect, which depends on the mean pore size and the mean free path of the gas molecules [38]. Given this dependence, the model describes the mesogeometry of the porous wall and the particulate layer structure as a packed bed of spherical particles [32], which is the most extended approach in the literature to analyse filtration efficiency [47] and pressure drop [15]. Accordingly, the permeability of any porous medium is determined as

$$
k=f(\varepsilon) d_{c}^{2} S C F,
$$

where $\varepsilon$ is the porosity, $f(\varepsilon)$ is a function of Kuwabara's hydrodynamic factor [48] and $d_{c}$ is the mean collector diameter, which is a function of the porosity and mean pore diameter. Finally SCF represents the Stokes-Cunningham factor, which accounts for the slip-flow effect and corrects the permeability as a function of the flow properties.

The model was applied here to loading conditions assuming the following hypotheses and criteria to distribute the collected soot:

1. Firstly, the soot was distributed inside the porous wall until the saturation soot mass was reached (deep bed filtration regime) and then the particulate layer was formed (cake bed filtration regime). 
2. The penetration of soot inside the porous wall was only partial, as discussed in both experimental [49] and modelling [50] studies. Therefore, the porous wall was divided into two regions: a layer with soot deposition, in which the porosity and the mean pore diameter are reduced, followed by a layer which is assumed to be kept fully clean. Therefore, the effective porous wall permeability $\left(k_{w, e}\right)$ was computed as [32]:

$$
k_{w, e}=\frac{k_{w} k_{w_{0}}}{f_{w, s a t} k_{w_{0}}+\left(1-f_{w, s a t}\right) k_{w}}
$$

In Eq. (8), $k_{w_{0}}$ is the clean porous wall permeability, $k_{w}$ is the loaded porous wall permeability and $f_{w, s a t}$ is the fraction of porous wall filled with soot. The porosity and the mean pore diameter of the loaded porous wall were calculated making use of the shape factor concept to account for the growth of the collector unit diameter governing pressure drop [32] and filtration efficiency [51] phenomena.

3. The soot mass forming the particulate layer was distributed uniformly along the inlet channel length. Therefore, the thickness of the particulate layer was assumed to be constant.

4. The permeability of the particulate layer was calculated imposing the collector unit diameter equal to the mode of the particle size distribution during the loading process $(69 \mathrm{~nm})$ and by the porosity, which was set to 0.6 [32]. The porosity of the particulate layer defines the density as

$$
\rho_{p l}=\rho_{C}\left(1-\varepsilon_{p l}\right)
$$

so that the particulate layer thickness $\left(w_{p l}\right)$ for a given amount of soot mass in the particulate layer was obtained according to:

$$
w_{p l}=\frac{\alpha-\sqrt{\alpha^{2}-\frac{m_{p l}}{N_{i n} L_{e} \rho_{p l}}}}{2}
$$

In Eq. (10), $m_{p l}$ and $\rho_{p l}$ represent the soot mass and density of the particulate layer respectively; $N_{i n}$ is the number of inlet channels and $L_{e}$ is the effective length of the inlet channels.

\section{Analysis of the transient response}

The modelling of the different tests shows that the approach for unsteady compressible flow together with a numerical solution based on shock-capturing methods provides an accurate description of pressure wave propagation. Experimental and modelled results corresponding to impulse flow tests with clean and loaded DPF are compared in Figure 11. As previously explained, the modelling of these tests was performed based on single channel calculations. Despite this simplification and the assumptions derived from the unknown soot mass distribution, the model is clearly able to reproduce the DPF dynamic response in clean conditions as well as the variations due to soot loading. The model perfectly catches the reflected wave under clean DPF conditions, in both the expansion and diffusion pulse phases. However, the peak of the reflected pulse under soot loading conditions appears to be slightly shifted. It can be 
explained by the high thickness of soot accumulated at the rear end of the inlet channel, which reduces the effective length.

The transmitted wave is accurately modelled, as it can be observed in Figure 11(b). In this case, modelled results for clean and highly loaded DPF cases show very good agreement with the experimental data, what indicates the model ability to predict the pressure drop. Based on these results, several soot loadings were explored to identify how the DPF response is progressively modified. The results of this study are presented in Figure 12. Two intermediate soot loading levels were considered between the tested cases. On one hand, the case of porous wall saturation ( $0.36 \mathrm{~g} / \mathrm{l})$ shows a minor influence on the transmission. Nevertheless, the decrease of the porous wall permeability gives rise to a more abrupt recuperation of the compression in the reflected wave. Once the particulate layer is formed, which corresponds to the $10 \mathrm{~g} / 1$ and $22 \mathrm{~g} / 1$ cases, the reflection gets clearly dominated by the low porous substrate permeability and the decrease in the volume of the inlet channels. The transmitted pressure is also highly affected by the low permeability of the particulate layer as well as by the change in the particulate layer thickness.

The frequency domain results obtained from the model by imposing constant particulate layer thickness along the channel length are shown in Figure 13 for a pressure excitation amplitude of 200 mbar. The prediction of the reflected spectrum is very precise up to $1000 \mathrm{~Hz}$. The model reproduces satisfactorily the change in SPL due to soot loading variations in the whole range. The trough observed in the reflected SPL close to $500 \mathrm{~Hz}$ in clean conditions is reproduced with a slight shift. When the DPF is loaded, this trough gets smoothed in the experimental results and shifted towards a higher frequency $(700 \mathrm{~Hz})$. However, the model predicts a much more marked SPL decrease with a noticeable frequency mismatch, since the peak is moved to $540 \mathrm{~Hz}$. This shift in the location of the reactive response when the DPF is highly loaded is more evident in the analysis of the transmitted pressure. The model prediction is fairly accurate under clean conditions for both SPL and transmission loss, which show an essentially flat spectrum. When the DPF gets loaded the model again provides a very good description of the SPL and the transmission loss up to $1000 \mathrm{~Hz}$, but the reactive response of the DPF device is missed. The experimental results indicate the existence of reactive characteristics located at $300 \mathrm{~Hz}$ and at a band from $700 \mathrm{~Hz}$ to $1000 \mathrm{~Hz}$. However, the model predicts a peak in the transmission loss at $500 \mathrm{~Hz}$.

As described in Section 3, a slight dome also appears in the experimental transmitted SPL around $500 \mathrm{~Hz}$ (valley in the transmission loss). Therefore, the fact that the model preserves this frequency under soot loading conditions should be expected since the effective length of the system was not modified with respect to clean conditions. However, the very high soot loading at which the tests were performed might indicate that some soot deposits were moved to the rear end of the inlet channels, so that the effective length was shortened. Figure 14 shows the modelled results once the effective length of the DPF inlet channels was reduced from $0.2 \mathrm{~m}$ to $0.17 \mathrm{~m}$. With this geometry variation the model response is clearly improved and agrees with the experimental data. In the case of the reflected SPL, the decreasing peak is now located at $750 \mathrm{~Hz}$ thus reducing the mismatch with respect to the experimental results. The best improvements are seen in the prediction of the transmitted pressure. Although the reactive peak at $300 \mathrm{~Hz}$ is still missed, the dissipative-like behaviour is reproduced up to $800 \mathrm{~Hz}$. At this frequency, the modelled response is 
smoother than in the experiments, but the pass-band located between $770 \mathrm{~Hz}$ and $950 \mathrm{~Hz}$ in the experimental results is predicted.

The ability to reproduce the dynamic response of the DPF was also checked under engine-like operating conditions. Figure 15 shows the comparison between experimental and modelled results in the time domain corresponding to tests in the unsteady flow test rig. As explained in Section 4, note that a single channel was considered in the model for cold conditions, whereas radial discretization (10 channel beams) was used in the case of hot flow to properly account for the influence of heat transfer [31]. The operating points correspond to those previously shown in Figure 5. The model exhibits good agreement with the experimental data for both the reflected and transmitted mean pressure. The duration and amplitude of the pulses is also modelled with accuracy indicating the model sensitivity to changes in the mean mass flow, gas temperature and amplitude of the inlet pressure pulse. Only a slight shift is noticed in the case of the transmitted pressure at high mass flow and low gas temperature.

The model was then applied to all operating points tested in order to check its capability to reproduce the trends observed in the experimental data. Figures 16 and 17 show, respectively, the modelled reflected and transmitted SPL corresponding to the second harmonic. Comparison with the experimental data shown in Figures 7 and 8 reveals that the model is sensitive to the effect of the different flow properties as well as to DPF soot loading. The increase in the SPL with pulse amplitude and its decrease as the DPF gets loaded is properly predicted. Also, the minor influences of gas temperature and pulse frequency are observed in the model results.

As a summary of the modelled transient response, Figure 18 represents the deviation in dB between experiment and model for the reflected and transmitted SPL, up to the 12th order harmonic. It can be observed that this deviation falls within a band of $\pm 5 \mathrm{~dB}$ for most of the harmonics in all the operating points. Despite the fact that the low orders of some operating points are falling out of this band, the general trend is that the deviation and the dispersion increase with frequency, i.e. at high orders. In the reflected SPL the highest error is clearly affecting to the 12th order harmonic, especially at low temperature conditions. This is also observed in the case of the transmitted SPL, for which only some 12th and 10th order harmonics lie out of the threshold band.

\section{Summary and conclusions}

An analysis of the transient response of wall-flow DPFs has been presented. This has been based on a combined experimental and computational approach covering variations in flow properties as well as changes in the monolith substrate by considering extreme porous medium conditions, from clean to highly soot loaded.

The flow characteristics were controlled in two experimental facilities. The DPF was firstly excited with isolated pressure pulses without mean flow. The pulse amplitude and duration were varied. The pulse characteristics swept showed a scaled change in reflection and transmission in the time and the frequency domains for a given soot loading. However, soot loading variation influences both transmission, by means of the pressure drop increase as soot accumulates, and reflection. The reduction of the permeability results in the change of the reflected wave pattern from a 
dominant open end reflection to a closed-end-like compression propagating back in the upstream direction. In terms of SPL, the reflection slightly increases its level while the transmission clearly decreases. Nevertheless, the dissipative behaviour of the DPF prevails on the reactive response, which essentially appears in the transmission as the DPF gets loaded.

Tests with periodic excitation and mean flow, both at low and high temperature, confirmed that the main effects of the soot loading variation appear on the pressure drop, which increases the engine back-pressure, but also in the reflection pattern. This multiple effect must be considered in any compromise solution for matching the device with upstream elements in the exhaust line. With respect to the influence of unsteady flow characteristics, the response is mainly sensitive to changes in pulse amplitude and soot loading. Both the reflected and transmitted SPL increase with the pulse amplitude, due to the higher instantaneous local velocity. Regarding the soot loading, its increase results in a decrease of the transmitted SPL with minor influence on reflection. Other variables such as mean mass flow, temperature or pulse frequency show negligible influence on the DPF response variation.

In order to analyze the observed response of wall-flow monoliths, a non-linear time-domain model was proposed. The model solves the governing equations in the monolith channels by means of sock capturing methods based on a 1D approach and assuming compressible unsteady flow. Quasi-steady flow is assumed across the porous medium and non-inertial pressure drop contributions are modelled as boundary conditions in the inlet and outlet canning volumes. This allows a proper management of time derivatives and space gradients in flow properties, which become important as pulse amplitude increases, as in the case of high mass flow or in pre-turbo DPF applications. The model results showed good agreement with the experimental data in both the time and the frequency domains demonstrating the sensitivity to variations in flow and substrate properties. The model reproduces with high accuracy the dissipative characteristics of the transient response, but some limitations in the description of reactive features arise. These can be attributed to the $0 \mathrm{D}$ approach used to describe the inlet and outlet volumes, and the simplifications required to describe the distribution of soot deposits. It has been shown that under very high soot loading levels, soot deposits at the rear end of the inlet channels play a major role in the reactive response, as indicated by the need to consider a corrected effective length in the model. Additionally, the consideration of the porous substrate as an homogeneous medium is shown to be essentially valid, as it provides an accurate prediction of the DPF transient response in the frequency domain up to the 10th harmonic for a wide range of engine operating conditions.

\section{Acknowledgements}

This work has been partially supported by the Spanish Ministerio de Economía y Competitividad through Grant No. TRA2013-40853-R.

[1] Regulation (EC) no 692/2008 of 18 July 2008 implementing and amending regulation (EC) no 715/2007 of the European Parliament and of the Council on type-approval of motor vehicles with respect to emissions from light passenger and commercial vehicles (Euro 5 and Euro 6) and on access to vehicle repair and maintenance information, Official Journal of the European Union (July 2008). 
[2] A. Mamakos, G. Martini, P. Dilara, Y. Drossinos, Feasibility of introducing particulate filters on gasoline direct injection vehicles. A cost benefit analysis, JRC Scientific and Policy Reports EUR 25297, 2011.

[3] A. Mamakos, N. Steininger, G. Martini, P. Dilara, Y. Drossinos, Cost effectiveness of particulate filter installation on direct injection gasoline vehicles, Atmos. Environ. 77 (2013) 16-23.

[4] C.D. Depcik, A.J. Hausmann, Review and a methodology to investigate the effects of monolithic channel geometry, J. Eng. Gas Turb. Power 135 (2013) $0323011-16$.

[5] C. Benaqqa, M. Gomina, A. Beurotte, M. Boussuge, et al., Morphology, physical, thermal and mechanical properties of the constitutive materials of diesel particulate filters, Appl. Therm. Eng. 62 (2014) 599-606.

[6] O. Lefort, J. Herreros, A. Tsolakis, The use of a partial flow filter to assist the diesel particulate filter and reduce active regeneration events, SAE Int. J. Engines 7(4) (2014) 1953-1960.

[7] M. Murtagh, Diesel particulate filters (DPF): A short course, in: Diesel particulate and NOx emissions course, University of Leeds, Ann Arbor, MI, 2002.

[8] O. Salvat, P. Marez, G. Belot, Passanger car serial application of a particulate filter system on a common-rail, direct-injection diesel engine, in: SAE Technical Paper 2000-01-0473, 2000.

[9] E. Ohara, Y. Mizuno, Y. Miyairi, T. Mizutani, et al. Filtration behavior of diesel particulate filters (1), in: SAE Technical Paper 2007-01-0921, 2007.

[10] F. Millo, M. Andreata, M. Rafigh, D. Mercuri, C. Pozzi, Impact on vehicle fuel economy of the soot loading on diesel particulate filters made of different substrate materials, Energy 85 (2015) 19-30.

[11] C. Beatrice, S. Di Iorio, C. Guido, P. Napolitano, Detailed characterization of particulate emissions of an automotive catalyzed DPF using actual regeneration strategies, Exp. Therm. Fluid Sci. 39 (2012) 45-53.

[12] A. Kotrba, T.P. Gardner, L. Bai, A. Yetkin, Passive regeneration response characteristics of a DPF system, in: SAE Technical Paper 2013-04$08,2013$.

[13] J. Song, J. Wang, A.L. Boehman, The role of fuel-borne catalyst in diesel particulate oxidation behavior, Combust. Flame 146 (2006) $73-84$.

[14] J.R. Serrano, H. Climent, P. Piqueras, E. Angiolini, Analysis of fluid-dynamic guidelines in diesel particulate filter sizing for fuel consumption reduction in post-turbo and pre-turbo placement, Appl. Energ. 132 (2014) 507-523.

[15] T. Bollerhoff, I. Markomanolakis, G. Koltsakis, Filtration and regeneration modeling for particulate filters with inhomogeneous wall structure, Catal. Today 188 (2012) 24-31.

[16] V. Bermúdez, J.R. Serrano, P. Piqueras, O. García-Afonso, Analysis of heavy-duty turbocharged diesel engine response under cold transient operation with a pre-turbo aftertreatment exhaust manifold configuration, Int. J. Engine Res. 14 (4) (2013) $341-353$.

[17] V. Bermúdez, J.R. Serrano, P. Piqueras, O. García-Afonso, Pre-DPF water injection technique for pressure drop control in loaded wall-flow diesel particulate filters, Appl. Energ. 140 (2015) 234-245.

[18] J.M. Lújan, V. Bermúdez, P. Piqueras, O. García-Afonso, Experimental assessment of pre-turbo aftertreatment configurations in a single stage turbocharged diesel engine. Part 1: Steady-state operation, Energy 80 (2015) 599-613.

[19] A. Sappok, V. Wong, Ash effects on diesel particulate filter pressure drop sensitivity to soot and implications for regeneration frequency and DPF control, SAE Int. J. Fuels. Lubr. 3 (2010) 380-96.

[20] K. Ogyu, K. Ohno, S. Hong, T. Komori, Ash storage capacity enhancement of diesel particulate filter, in: SAE Technical Paper 2004-01-0949, 2004.

[21] S. Allam, M. Åbom, Acoustic modelling and testing of diesel particulate filters, J. Sound Vib. 288 (2005) $255-273$.

[22] X. Hua, J. Liu, D. Herrin, T. Elnady, Numerical simulation of diesel particulate filters in exhaust systems, in: SAE Technical Paper 2011-01$1559,2011$.

[23] A. Katari, M. Syed, M. Sickels, T. Wahl, S. Rajadurai, Effect of aspect ratio on pressure drop and acoustics in diesel particulate filters, in: SAE Technical Paper 2004-01-0695, 2004. 
[24] J. Hicks, W. Hill, A. Kotrba, DPF acoustic performance: an evaluation of various substrate materials and soot conditions, in: SAE Technical Paper 2011-01-2198, 2011.

[25] R. Glav, H. Boden, M. Åbom, An acoustic model for automobile catalytic converters, in: Proceedings Inter-Noise Conference, 1988, pp. 1261-1266.

[26] S. Allam, M. Åbom, On acoustic modeling and testing of diesel particulate filters, in: Proceedings of Inter-Noise Conference, Vol. $250,2002$.

[27] S. Allam, M. Åbom, Sound propagation in an array of narrow porous channels with application to diesel particulate filters, J. Sound Vib. 291 (2006) 882-901.

[28] W. Gao, L. Feng, FEM analysis on acoustic performance of wall flow diesel particulate filters, Chin. J. of Mech. Eng. 24 (2011) 1-6.

[29] X. Hua, D.W. Herrin, T.W. Wu, T. Elnady, Simulation of diesel particulate filters in large exhaust systems, Appl. Acoust. 74 (2013) 13261332.

[30] A.J. Torregrosa, J.R. Serrano, F.J. Arnau, P. Piqueras, A fluid dynamic model for unsteady compressible flow in wall-flow diesel particulate filters, Energy 36 (2011) 671-684.

[31] J. Galindo, J.R. Serrano, P. Piqueras, O. García-Afonso, Heat transfer modelling in honeycomb wall-flow diesel particulate filters, Energy 43 (2012) 201-213.

[32] J.R. Serrano, F.J. Arnau, P. Piqueras, O. García-Afonso, Packed bed of spherical particles approach for pressure drop prediction in wall-flow DPFs (diesel particulate filters) under soot loading conditions, Energy 58 (2013) 644-654.

[33] F. Payri, J.M. Desantes, A. Broatch, Modified impulse method for the measurement of the frequency response of acoustic filters to weakly nonlinear transient excitations, J. Acoust. Soc. Am. 107 (2) (2000) 731-738.

[34] G. Piero, L. Vergara, J.M. Desantes, A. Broatch, Estimation of velocity fluctuation in internal combustion engine exhaust systems through beam forming techniques, Meas. Sci. Technol. 11 (2000) 1585-1595.

[35] M. Piumetti, B. van der Linden, M. Makkee, P. Miceli, D. Fino, N. Russo, S. Bensaid, Contact dynamics for a solidsolid reaction mediated by gas-phase oxygen: Study on the soot oxidation over ceria-based catalysts, Appl. Catal., B 199 (2016) 96-107.

[36] A.G. Konstandopoulos, M. Kostoglou, E. Skaperdas, E. Papaioannou, D. Zarvalis, E. Kladopoulou, Fundamental studies of diesel particulate filters: transient loading, regeneration and aging, in: SAE Technical Paper 2000-01-1016, 2000.

[37] A.G. Konstandopoulos, E. Skaperdas, M. Masoudi, Microstructural properties of soot deposits in diesel particulate traps, in: SAE Technical Paper 2002-01-1015, 2002.

[38] F. Payri, A. Broatch, J.R. Serrano, P. Piqueras, Experimental-theoretical methodology for determination of inertial pressure drop distribution and pore structure properties in wall-flow diesel particulate filters (DPFs), Energy 36 (2011) 6731-6744.

[39] J. Galindo, J.R. Serrano, F.J. Arnau, P. Piqueras, Description of a Semi-Independent Time Discretization methodology for a one-dimensional gas dyanmics model, J. Eng. Gas Turb. Power 131 (2009) 034504.

[40] OpenWAM website, CMT-Motores Térmicos (Universitat Politécnica de Valéncia). www.openwam.org (2015).

[41] P. Lax, B. Wendroff, Systems of conservation laws, Commun. Pur. Appl. Math. 17 (1964) 381-398.

[42] J.R. Serrano, F.J. Arnau, P. Piqueras, O. García-Afonso, Application of the two-step Lax\&Wendroff-FCT and the CE-SE method to flow transport in wall-flow monoliths, Int. J. Comput. Math. 91 (1) (2014) 71-84.

[43] J.M. Desantes, J.R. Serrano, F.J. Arnau, P. Piqueras, Derivation of the method of characteristics for the fluid dynamic solution of flow advection along porous wall channels, Appl. Math. Model. 36 (2012) 3134-3152.

[44] E.J. Bisset, Mathematical model of the thermal regeneration of a wall-flow monolith diesel particulate filter, Chem. Eng. Sci. 39 (7-8) (1984) $1233-1244$.

[45] A.G. Konstandopoulos, J.H. Johnson, Wall-flow diesel particulate filters - Their pressure drop and collection efficiency, in: SAE Technical Paper 890405, 1989.

[46] F. Dullien, Porous Media. Fluid Transport and Pore Structure, Academic Press, New York, 1992.

[47] P. Tandon, A. Heibel, J. Whitmore, N. Kekre, K. Chithapragada, Measurement and prediction of filtration efficiency evolution of soot loaded diesel particulate filters, Chem. Eng. Sci. 65 (2010) 4751-4760. 
[48] S. Kuwabara, The forces experienced by randomly distributed parallel circular cylinders or spheres in a viscous fluid at small Reynolds numbers, J. Phys. Soc. Jpn 14 (1959) 527-532.

[49] D. Fino, N. Russo, F. Millo, D.S. Vezza, et al., New tool for experimental analysis of diesel particulate filter loading, Top Catal 52 (2009) $13-20$.

[50] K. Yamamoto, A. Oohori, H. Yamashita, S. Daido, Simulation on soot deposition and combustion in diesel particulate filter, Proc. Combust. Inst. 32 (2009) 1965-1972.

[51] J.R. Serrano, H. Climent, P. Piqueras, E. Angiolini, Filtration modelling in wall-flow particulate filters of low soot penetration thickness, Energy 112 (2016) 883-898.

\section{Nomenclature}

\begin{tabular}{|c|c|}
\hline$d_{c}$ & collector unit diameter \\
\hline$e_{0}$ & specific stagnation internal energy \\
\hline$f_{w, s a t}$ & fraction of porous wall occupied by soot mass \\
\hline$F$ & cross-section area \\
\hline$F_{w}$ & momentum transfer coefficient for square channels \\
\hline$h_{0}$ & specific stagnation enthalpy \\
\hline$k$ & permeability \\
\hline$k_{p l}$ & particulate layer permeability \\
\hline$k_{w}$ & porous wall permeability \\
\hline$k_{w_{0}}$ & clean porous wall permeability \\
\hline$k_{w, e}$ & effective porous wall permeability \\
\hline$L_{e}$ & effective channel length \\
\hline$m_{p l}$ & soot mass in the particulate layer \\
\hline$N_{\text {in }}$ & number of inlet channels \\
\hline$p$ & pressure \\
\hline$q$ & heat per unit of time and mass \\
\hline$t$ & time \\
\hline$u$ & velocity \\
\hline$u_{w}$ & filtration velocity \\
\hline$w_{p l}$ & particulate layer thickness \\
\hline$x$ & axial dimension \\
\hline
\end{tabular}

\section{Greek letters}

$\alpha \quad$ honeycomb cell size

$\varepsilon \quad$ porosity 


$\begin{array}{ll}\mu & \text { dynamic viscosity } \\ \rho & \text { gas density }\end{array}$

$\begin{array}{ll}\text { Subscripts } & \\ 0 & \text { outlet channel } \\ 1 & \text { inlet channel } \\ c & \text { composed } \\ i n c & \text { incident } \\ j & \text { type of monolith channel } \\ p l & \text { referred to the particulate layer } \\ r f & \text { reflected } \\ w & \text { referred to the porous wall } \\ C & \text { carbon }\end{array}$

Abbreviations

2LW+FCT two-step Lax\&Wendroff method and flux-corrected transport technique

BEM boundary element method

DPF diesel particulate filter

FCT flux-corrected transport technique

FEM finite element method

MoC Method of Characteristics

TL transmission loss

SCF Stokes-Cunningham factor

SPL sound pressure level

\section{List of Figures}

- Figure 1.- Schematic setup of the impulse test rig.

- Figure 2.- (a) Setup of the flow gas stand. (b) Pressure components defining the pulsating flow in the gas stand.

- Figure 3.- Incident, reflected and transmitted pressure pulses as a function of the pulse amplitude and the DPF soot loading.

- Figure 4.- Reflected and transmitted SPL and transmission loss as a function of the pressure excitation and the DPF soot loading.

- Figure 5.- Experimental pressure wave decomposition as a function of the DPF soot loading for different pulsating operating conditions.

- Figure 6.- Experimental reflected and transmitted SPL as a function of the DPF soot loading for different pulsating operating conditions.

- Figure 7.- Experimental reflected SPL corresponding to second and fourth order harmonics as a function of the pulse frequency and amplitude accounting for the influence of the mass flow, temperature and DPF soot loading. 
- Figure 8.- Experimental transmitted SPL corresponding to second and fourth order harmonics as a function of the pulse frequency and amplitude accounting for the influence of the mass flow, temperature and DPF soot loading.

- Figure 9.- Scheme of the axial and radial discretisation of the DPF monolith for numerical resolution of the governing equations.

- Figure 10.- Inlet channel mass flow and filtration velocity profiles as a function of the DPF mass flow in steady flow.

- Figure 11.- Comparison between experimental and modelled reflected and transmitted pressure waves as a function of the pulse excitation and the DPF soot loading.

- Figure 12.- Modelling of the DPF soot loading effect on the reflected and transmitted pressure waves. Excitation of 200 mbar in amplitude and $20 \mathrm{~ms}$ in duration.

- Figure 13.- Comparison between experimental and modelled reflected and transmitted SPL and transmission loss as a function of the pulse excitation and the DPF soot loading. Excitation of 200 mbar in amplitude and $20 \mathrm{~ms}$ in duration.

- Figure 14.- Comparison between experimental and modelled reflected and transmitted SPL and transmission loss as a function of the pressure excitation and the DPF soot loading imposing a reduction of the effective length of the inlet channels $(0.17 \mathrm{~m})$. Excitation of $200 \mathrm{mbar}$ in amplitude and $20 \mathrm{~ms}$ in duration.

- Figure 15.- Comparison between experimental and modelled reflected and transmitted pressure waves as a function of the DPF soot loading for different pulsating operating conditions.

- Figure 16.- Modelled reflected SPL corresponding to second order harmonic as a function of the pulse frequency and amplitude accounting for the influence of the mass flow, temperature and DPF soot loading.

- Figure 17.- Modelled transmitted SPL corresponding to second order harmonic as a function of the pulse frequency and amplitude accounting for the influence of the mass flow, temperature and DPF soot loading.

- Figure 18.- Error in the prediction of reflected and transmitted SPL as a function of pulse amplitude and frequency, mass flow, temperature, DPF soot loading and order of the harmonics. 
Table 1: Geometrical characteristics of the DPF.

\begin{tabular}{lcc}
\hline \hline Length & {$[\mathrm{m}]$} & 0.2 \\
Diameter & {$[\mathrm{m}]$} & 0.132 \\
Plug length & {$[\mathrm{mm}]$} & 3.2 \\
Wall permeability & {$\left[\mathrm{m}^{2}\right]$} & $2.49 \times 10^{-13}$ \\
Porosity & {$[\%]$} & 41.5 \\
Mean pore diameter & {$[\mu \mathrm{m}]$} & 12 \\
Cell density & {$[\mathrm{cpsi}]$} & 200 \\
Filter cell size & {$[\mathrm{mm}]$} & 1.486 \\
Number of channels & {$[-]$} & 4246 \\
Filtration area & {$\left[\mathrm{m}^{2}\right]$} & 2.5 \\
Inlet cone volume & {$\left[\mathrm{cm}^{3}\right]$} & 500 \\
Outlet cone volume & {$\left[\mathrm{cm}^{3}\right]$} & 450 \\
\hline \hline
\end{tabular}

Table 2: Characteristics of the pressure sensors.

\begin{tabular}{|c|c|c|c|c|}
\hline & & Kistler 7001 & Kistler 7061C & Kistler 4045A5 \\
\hline Type & & Piezoelectric & Piezoelectric & Piezoresistive \\
\hline Range & [bar] & $0 \ldots .250$ & $0 \ldots 300$ & $0 \ldots 5$ \\
\hline Overload & [bar] & 350 & 350 & 12.5 \\
\hline Sensitivity & & 80 [pC/bar] & -92 [pC/bar] & $100[\mathrm{mV} / \mathrm{bar}]$ \\
\hline Linearity & [\% FSO] & $\leq \pm 0.8$ & $\leq \pm 0.3$ & $\leq \pm 0.3$ \\
\hline Temperature range & {$\left[{ }^{\circ} \mathrm{C}\right]$} & $-196 \ldots 350$ & $-20 \ldots 350$ & $0 \ldots 140$ \\
\hline
\end{tabular}

Table 3: Amplitude and duration of the pressure excitations generated in the impulse test rig.

\begin{tabular}{cc|ccccc}
\hline \hline & & \multicolumn{5}{|c}{ Duration [ms] } \\
& & 8 & 11 & 14 & 17 & 20 \\
\hline & 60 & $\times$ & - & - & - & - \\
& 95 & - & $\times$ & $\times$ & - & - \\
Amplitude & 130 & - & $\times$ & $\times$ & $\times$ & - \\
{$[$ mbar] } & 165 & - & - & $\times$ & $\times$ & - \\
& 200 & - & - & - & - & $\times$ \\
\hline \hline
\end{tabular}


Table 4: Test matrix with pulsating flow across the DPF. Definition of the soot loading, mass flow, temperature and pulse characteristics.

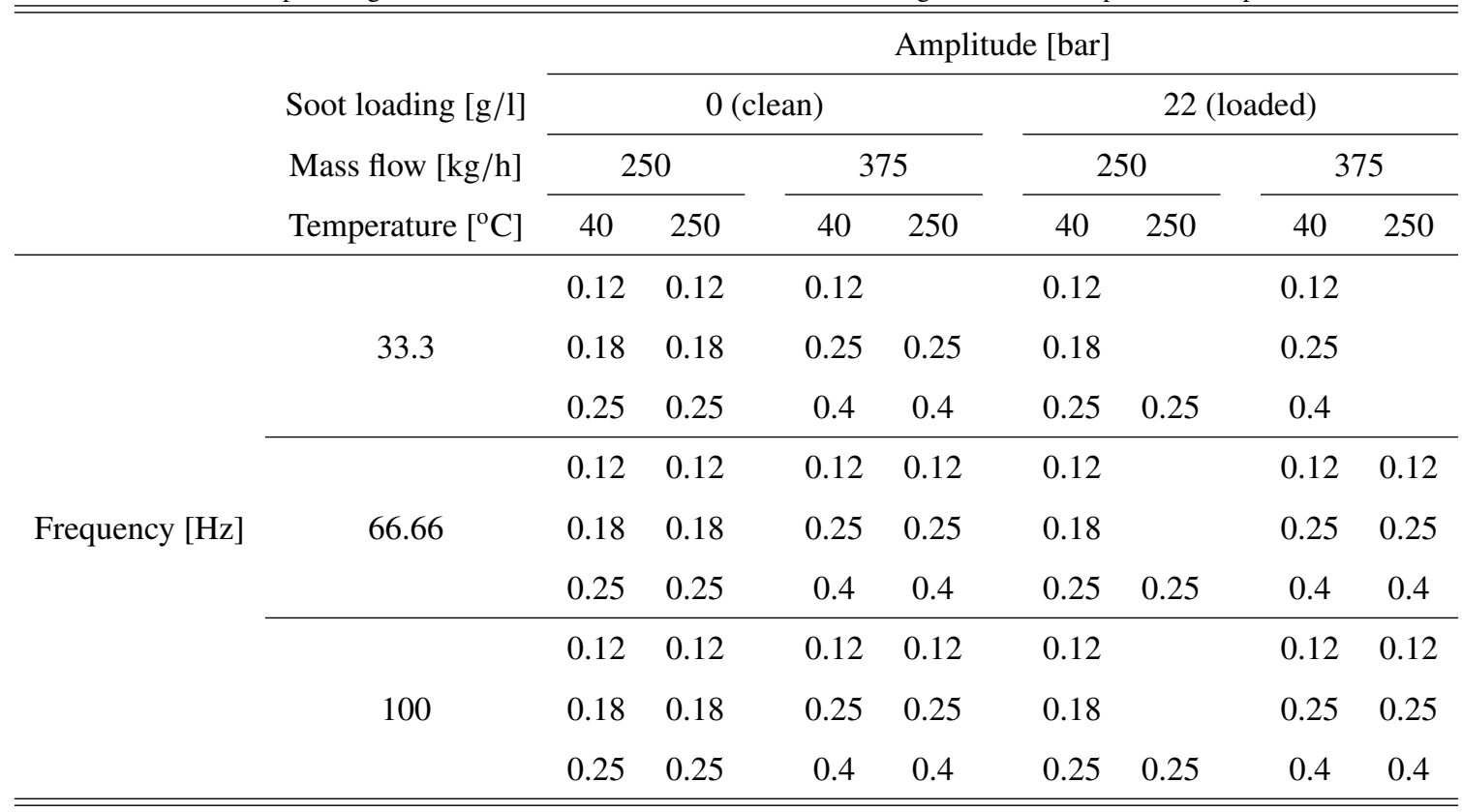

Table 5: Equivalence between the frequency of the pulses generated in the flow test rig and the speed of a four-stroke engine as a function of the number of cylinders.

\begin{tabular}{cccc}
\hline \hline Frequency [Hz] & \multicolumn{3}{c}{ Engine speed [rpm] } \\
& 2 cyl. & 3 cyl. & 4 cyl. \\
\hline 33.33 & 2000 & 1333 & 1000 \\
66.66 & 4000 & 2666 & 2000 \\
100 & 6000 & 4000 & 3000 \\
\hline \hline
\end{tabular}




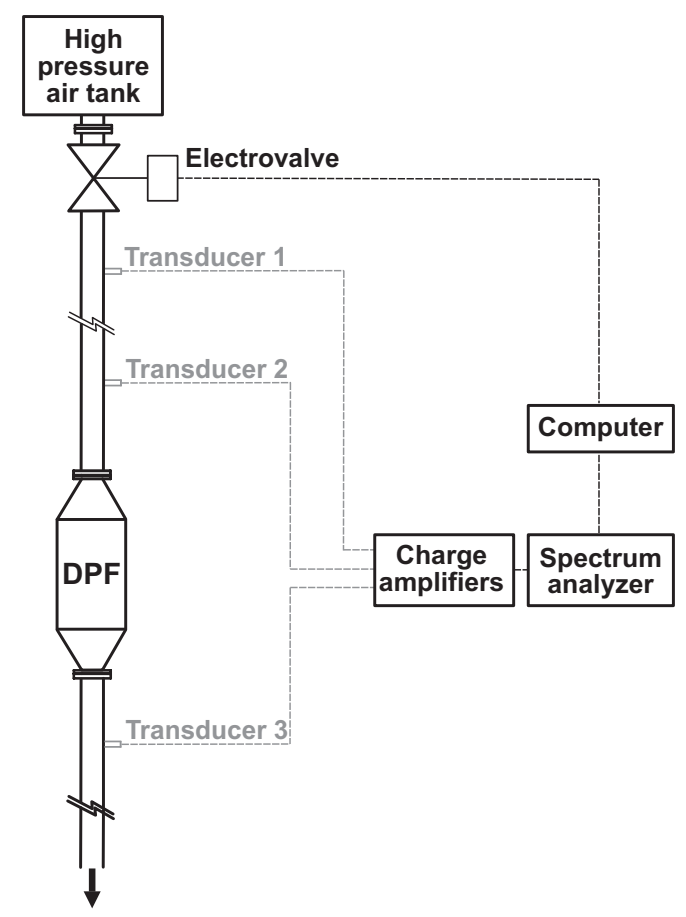

Figure 1: Schematic setup of the impulse test rig.

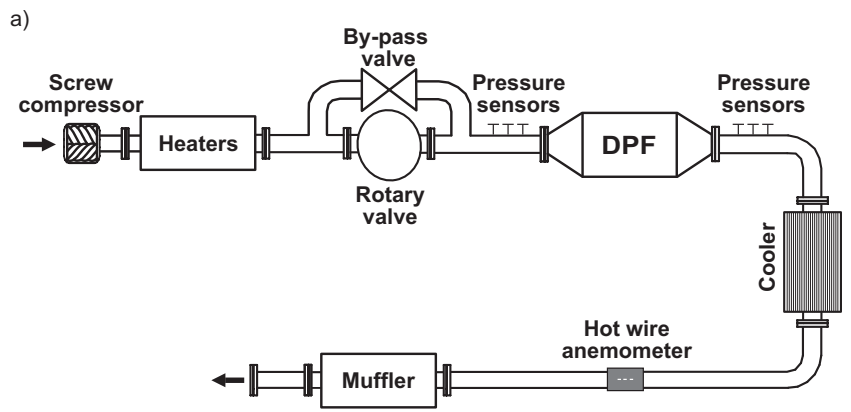

b)

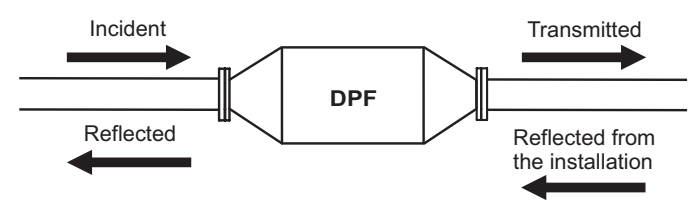

Figure 2: (a) Setup of the flow gas stand. (b) Pressure components defining the pulsating flow in the gas stand. 

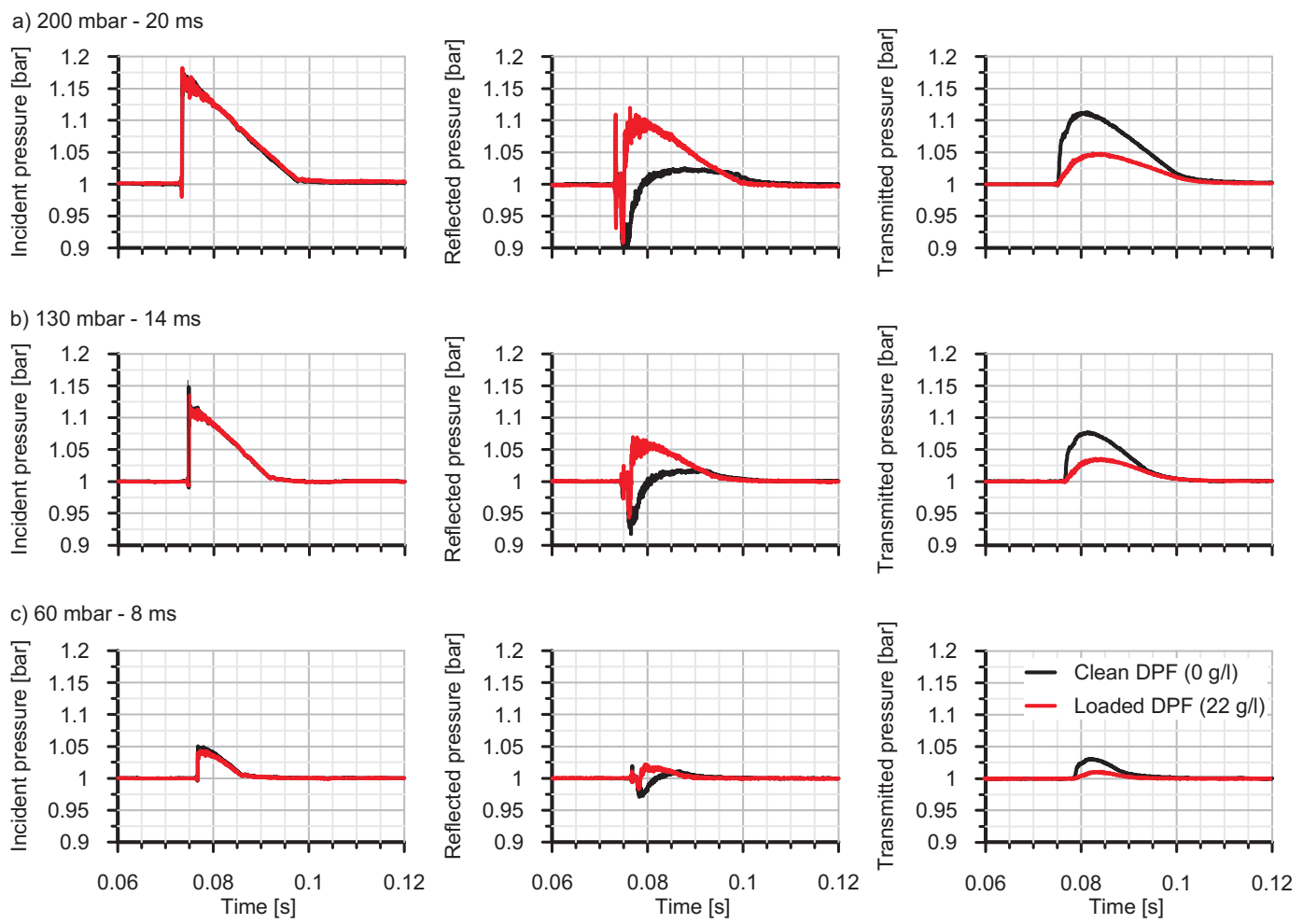

Figure 3: Incident, reflected and transmitted pressure pulses as a function of the pulse amplitude and the DPF soot loading. 
a) $200 \mathrm{mbar}-20 \mathrm{~ms}$
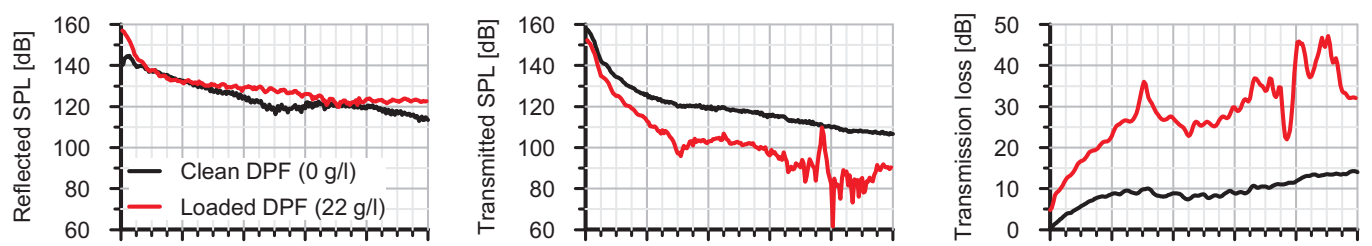

b) $130 \mathrm{mbar}-14 \mathrm{~ms}$
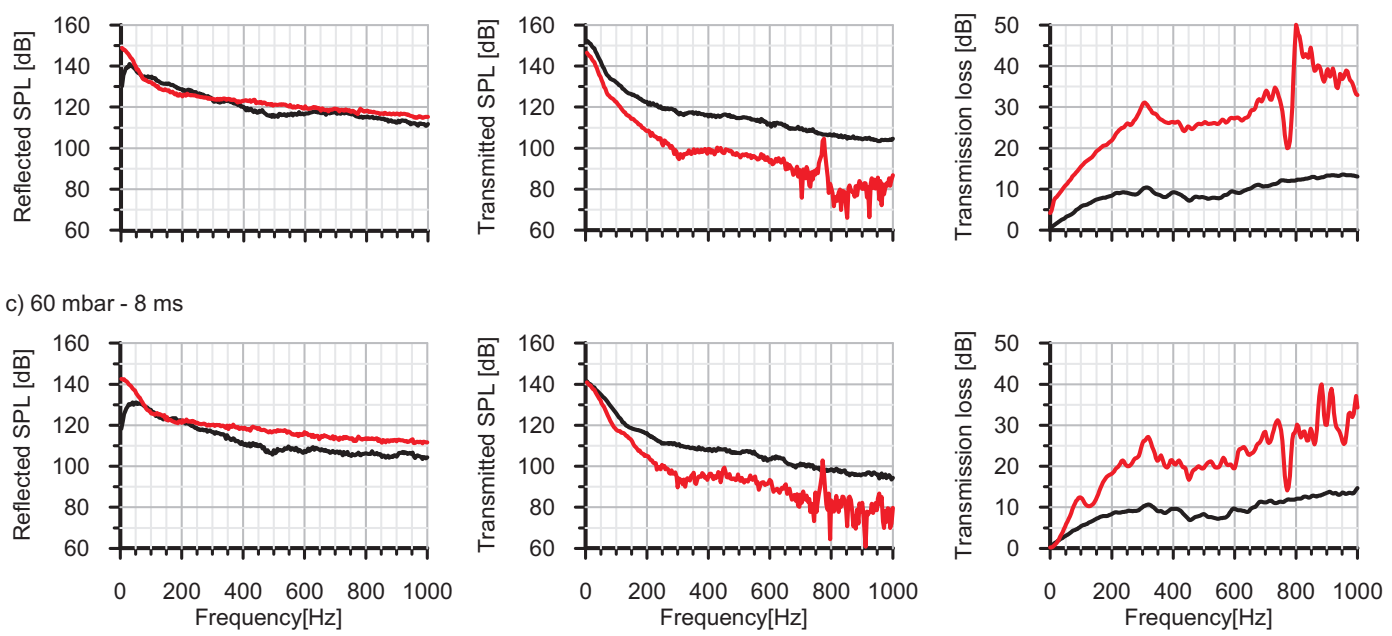

Figure 4: Reflected and transmitted SPL and transmission loss as a function of the pressure excitation and the DPF soot loading 

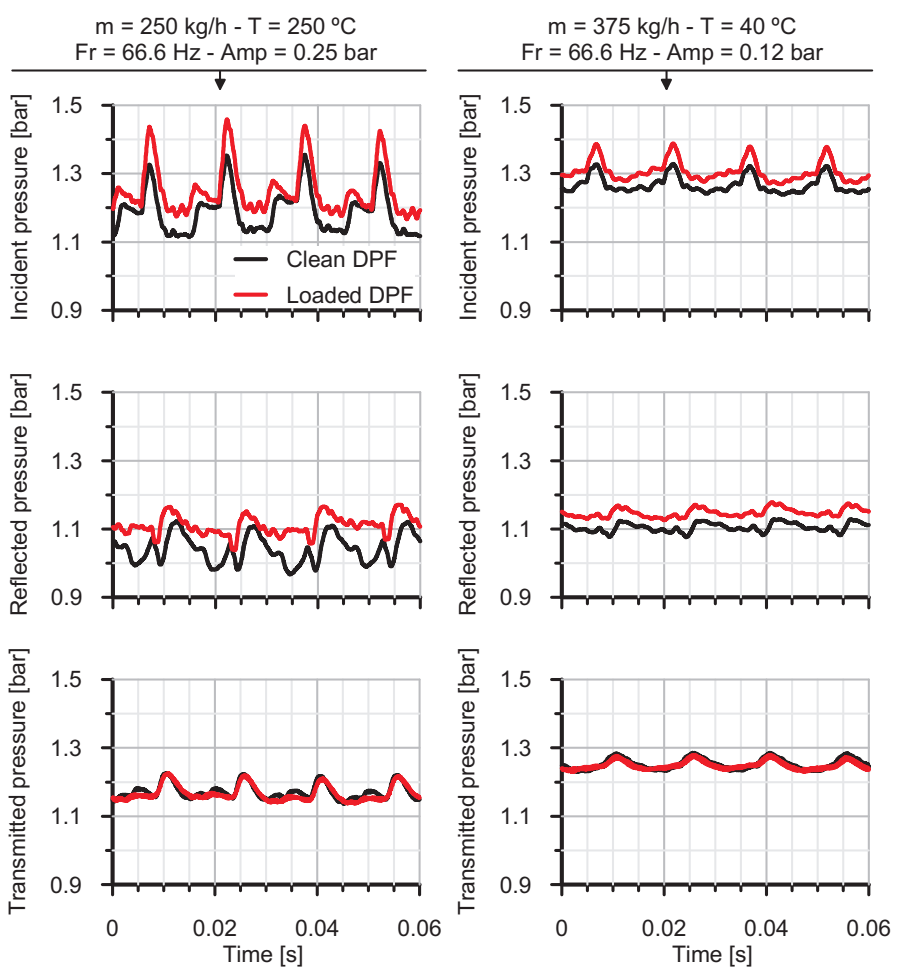

Figure 5: Experimental pressure wave decomposition as a function of the DPF soot loading for different pulsating operating conditions.
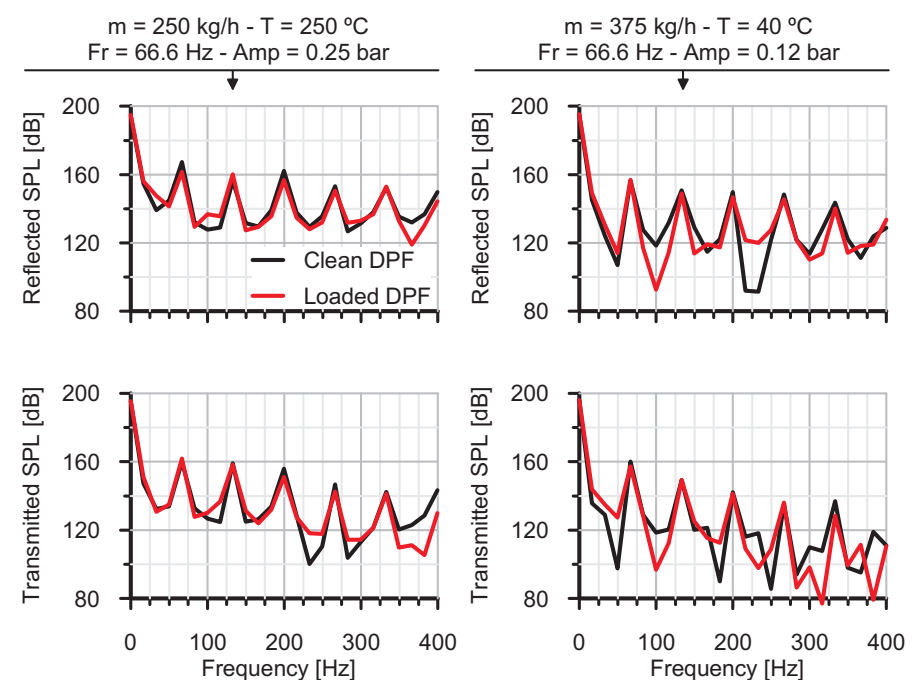

Figure 6: Experimental reflected and transmitted SPL as a function of the DPF soot loading for different pulsating operating conditions. 

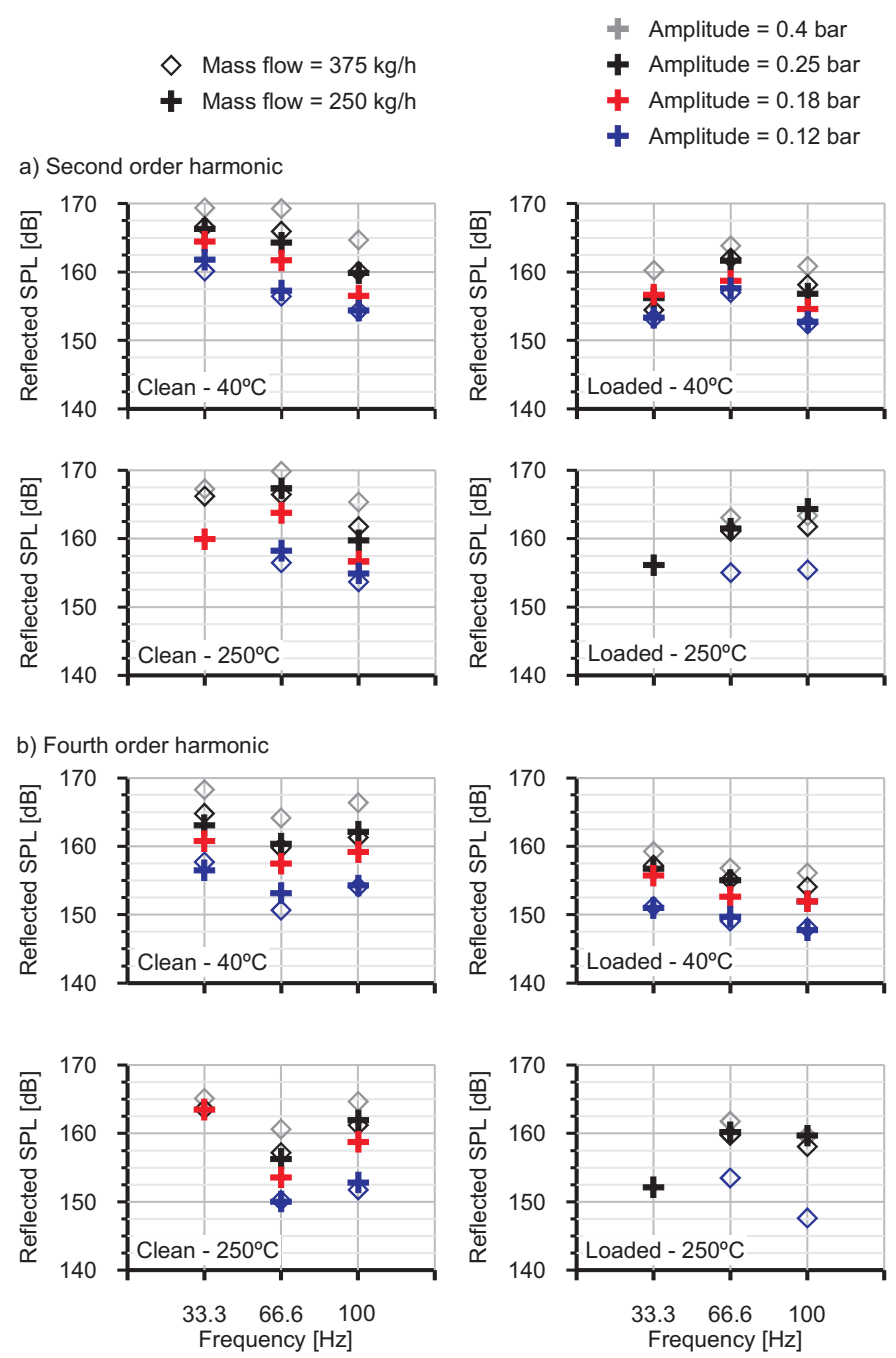

Figure 7: Experimental reflected SPL corresponding to second and fourth order harmonics as a function of the pulse frequency and amplitude accounting for the influence of the mass flow, temperature and DPF soot loading. 
$\diamond$ Mass flow $=375 \mathrm{~kg} / \mathrm{h}$

+ Mass flow $=250 \mathrm{~kg} / \mathrm{h}$

a) Second order harmonic
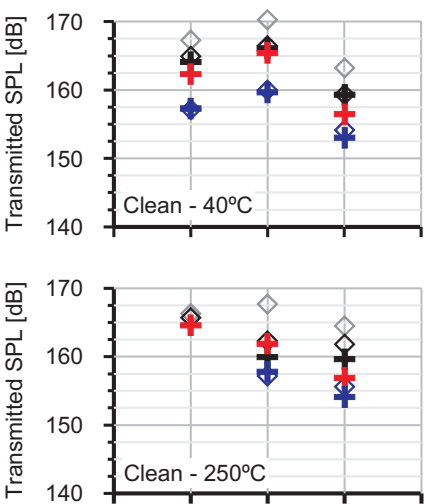

b) Fourth order harmonic
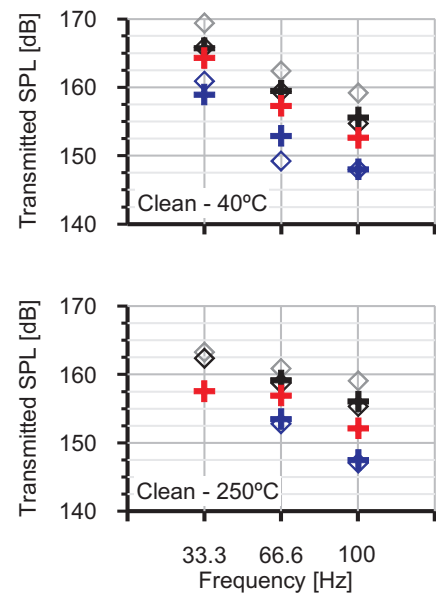

- Amplitude $=0.4$ bar

+ Amplitude $=0.25$ bar

+ Amplitude $=0.18 \mathrm{bar}$

+ Amplitude $=0.12$ bar
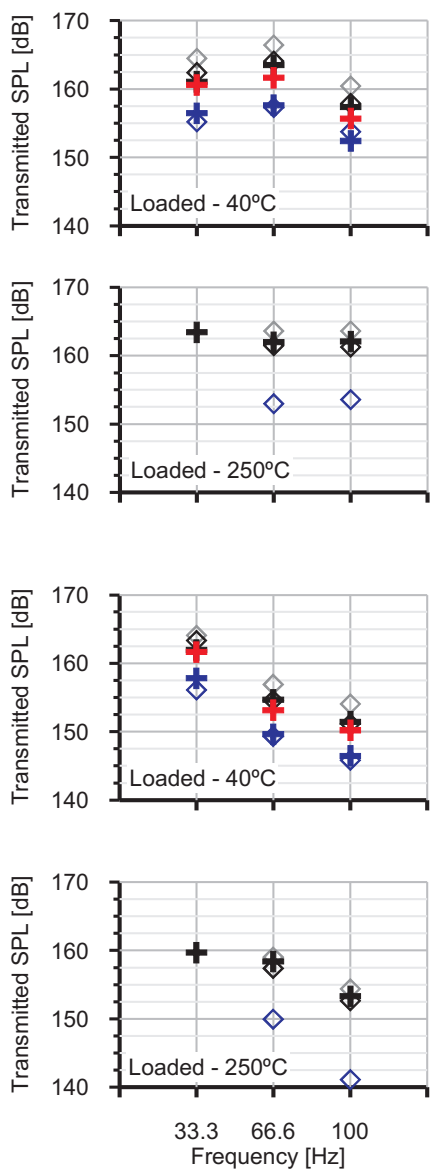

Figure 8: Experimental transmitted SPL corresponding to second and fourth order harmonics as a function of the pulse frequency and amplitude accounting for the influence of the mass flow, temperature and DPF soot loading.

- MoC $\quad 2 \mathrm{LW}+\mathrm{FCT}$

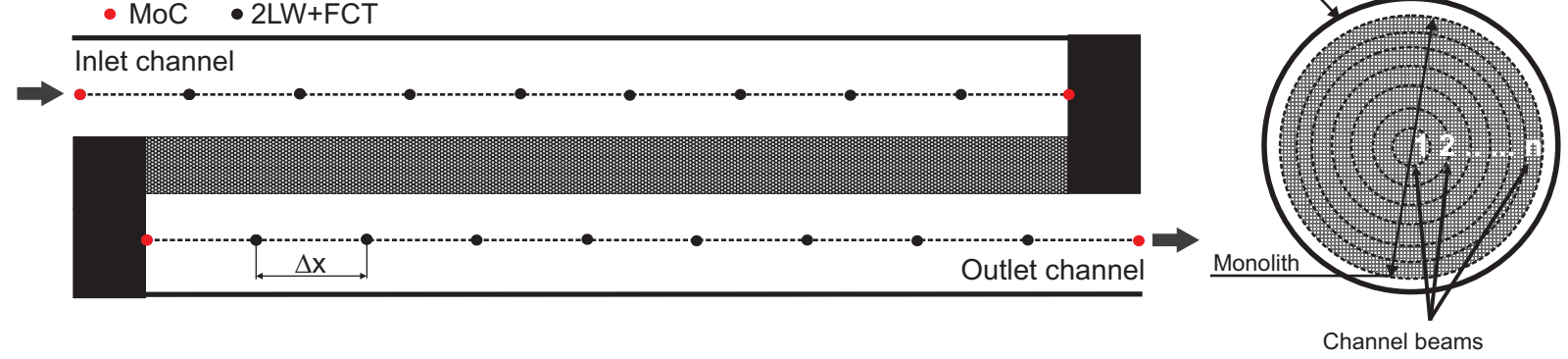

Figure 9: Scheme of the axial and radial discretisation of the DPF monolith for numerical resolution of the governing equations. 

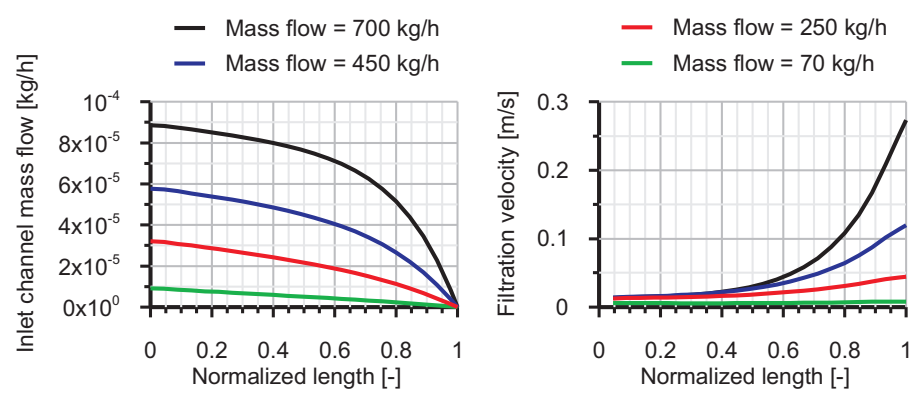

Figure 10: Inlet channel mass flow and filtration velocity profiles as a function of the DPF mass flow in steady flow.
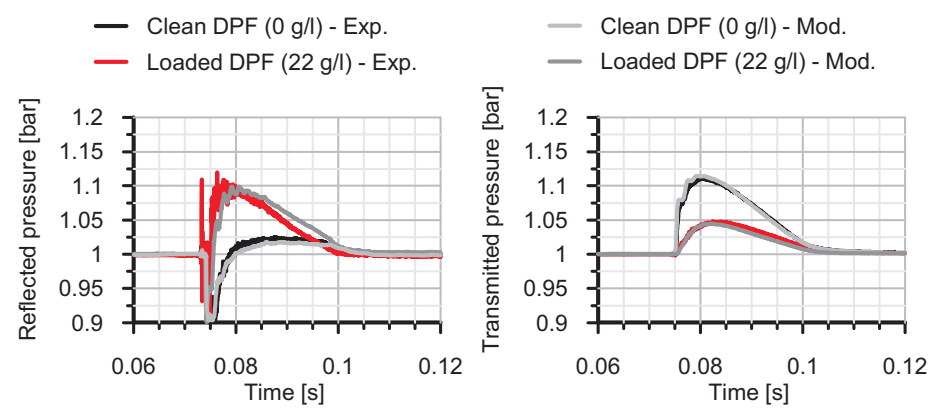

Figure 11: Comparison between experimental and modelled reflected and transmitted pressure waves as a function of the pulse excitation and the DPF soot loading.
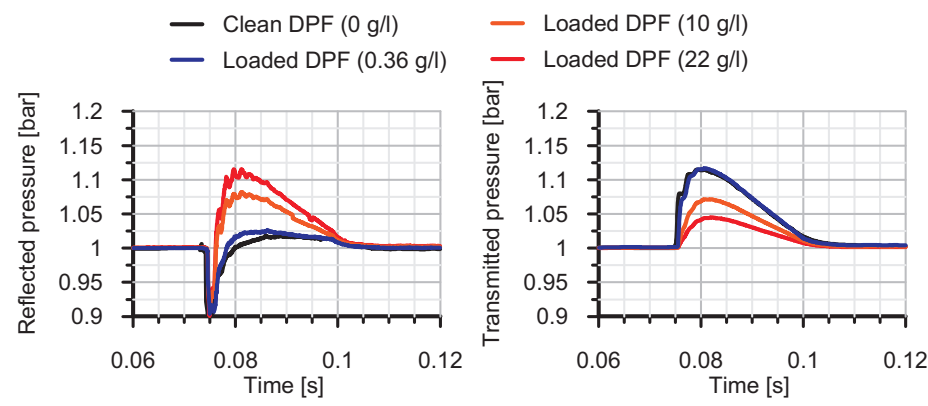

Figure 12: Modelling of the DPF soot loading effect on the reflected and transmitted pressure waves. Excitation of 200 mbar in amplitude and $20 \mathrm{~ms}$ in duration. 

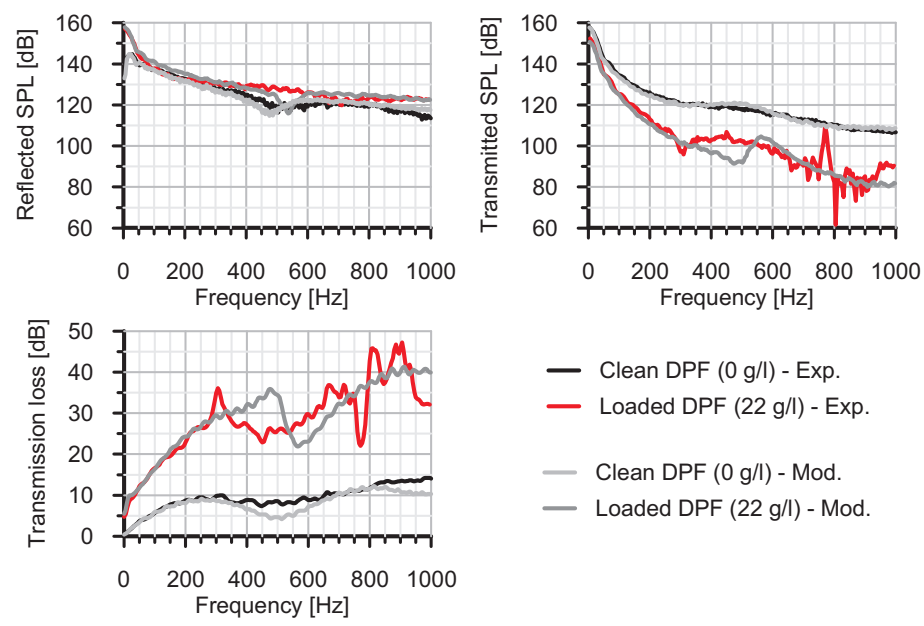

- Clean DPF $(0 \mathrm{~g} / \mathrm{l})$ - Exp.

- Loaded DPF (22 g/l) - Exp.

- Clean DPF (0 g/l) - Mod.

- Loaded DPF (22 g/l) - Mod.

Figure 13: Comparison between experimental and modelled reflected and transmitted SPL and transmission loss as a function of the pulse excitation and the DPF soot loading. Excitation of 200 mbar in amplitude and $20 \mathrm{~ms}$ in duration.
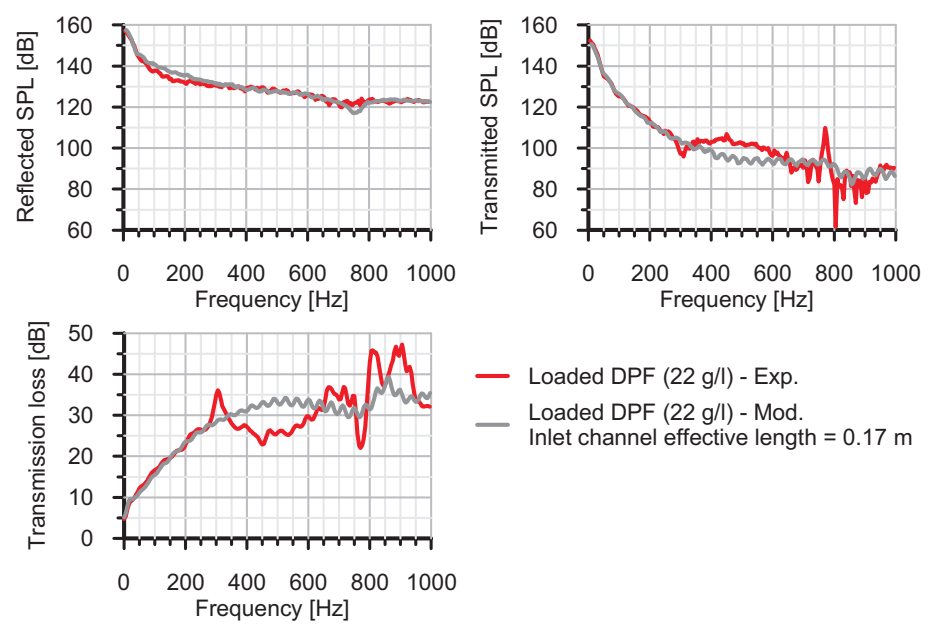

- Loaded DPF $(22 \mathrm{~g} / \mathrm{l})$ - Exp.

Loaded DPF $(22 \mathrm{~g} / \mathrm{l})$ - Mod

Inlet channel effective length $=0.17 \mathrm{~m}$

Figure 14: Comparison between experimental and modelled reflected and transmitted SPL and transmission loss as a function of the pressure excitation and the DPF soot loading imposing a reduction of the effective length of the inlet channels ( $0.17 \mathrm{~m})$. Excitation of 200 mbar in amplitude and $20 \mathrm{~ms}$ in duration. 


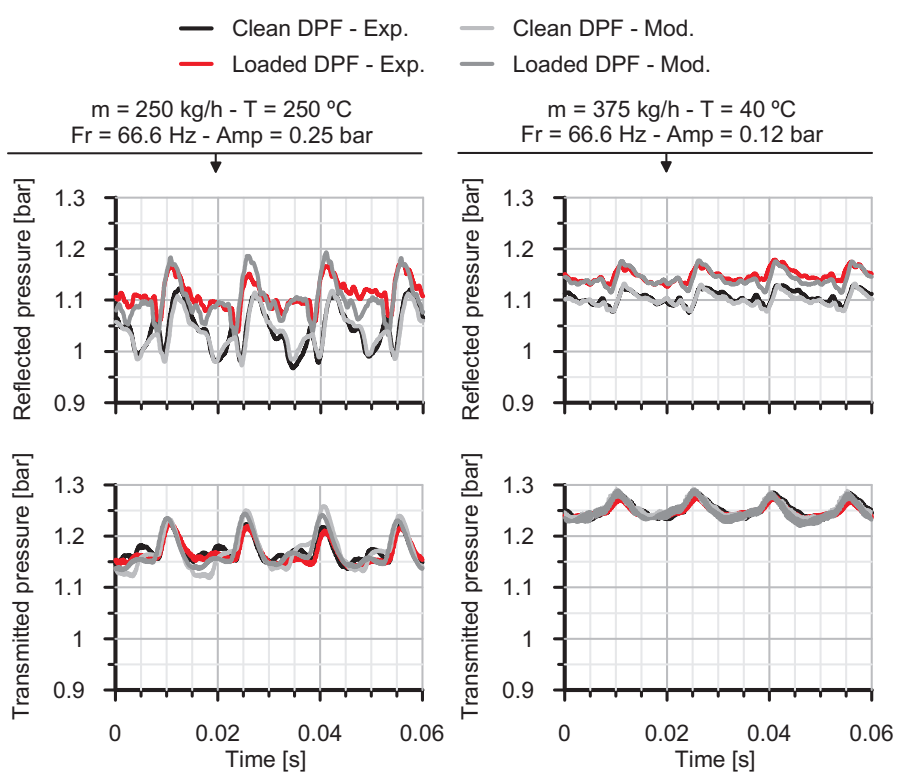

Figure 15: Comparison between experimental and modelled reflected and transmitted pressure waves as a function of the DPF soot loading for different pulsating operating conditions.

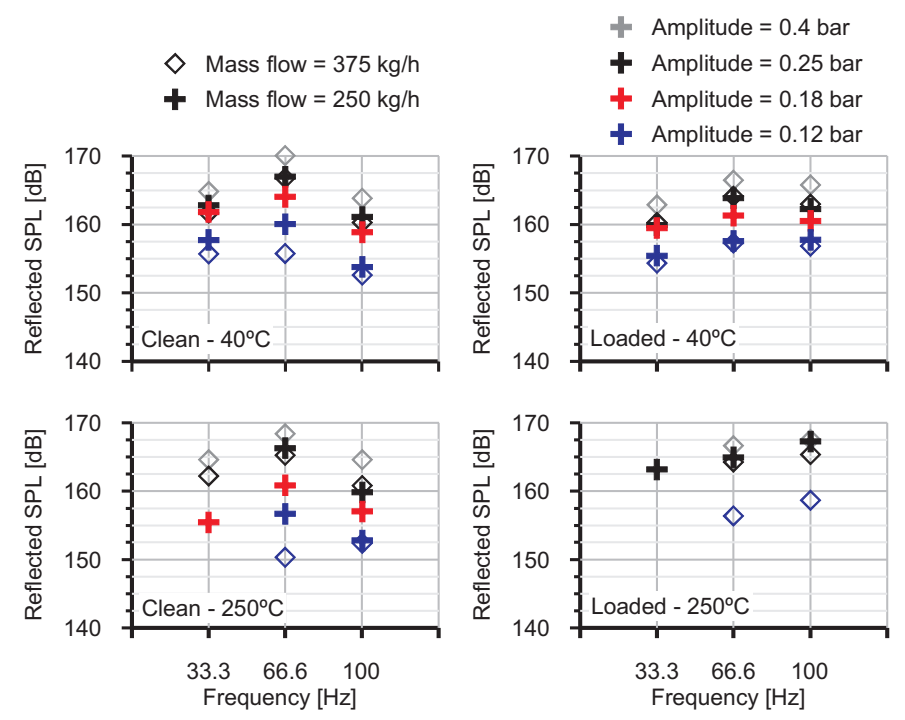

Figure 16: Modelled reflected SPL corresponding to second order harmonic as a function of the pulse frequency and amplitude accounting for the influence of the mass flow, temperature and DPF soot loading. 

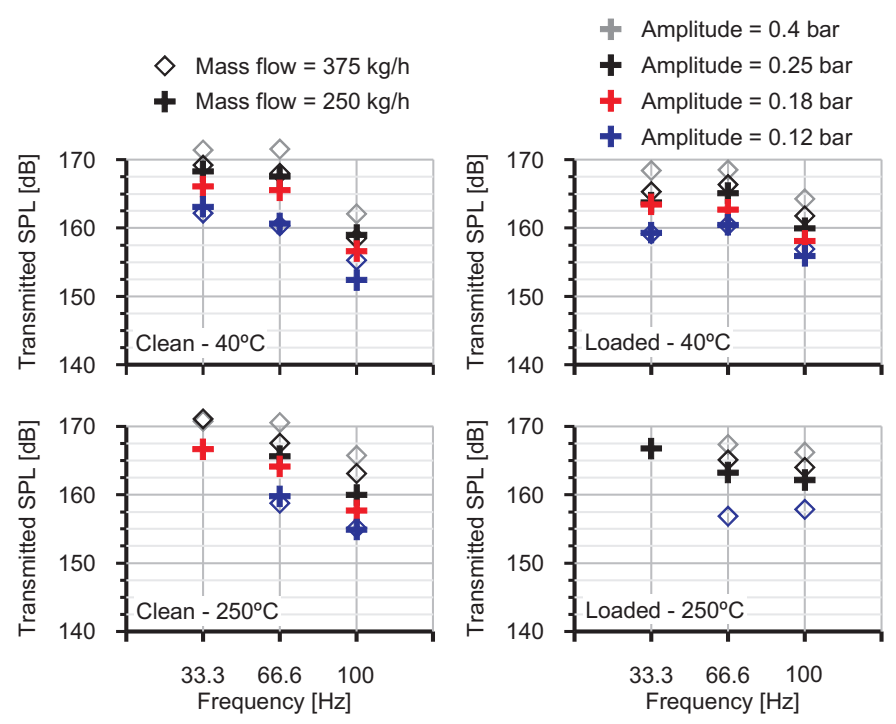

Figure 17: Modelled transmitted SPL corresponding to second order harmonic as a function of the pulse frequency and amplitude accounting for the influence of the mass flow, temperature and DPF soot loading. 
$\diamond$ Mass flow $=375 \mathrm{~kg} / \mathrm{h}$

4 Mass flow $=250 \mathrm{~kg} / \mathrm{h}$

a) Reflected SPL
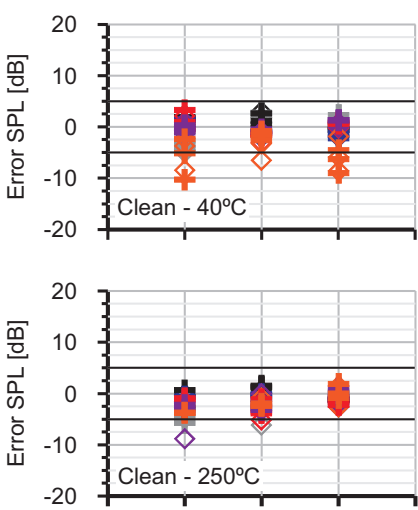

Transmitted SPL
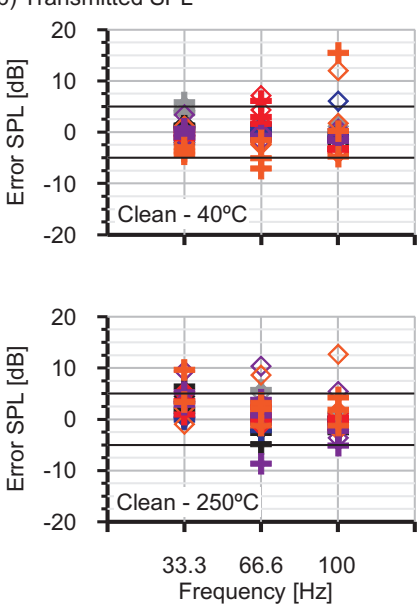

+ 2nd order +8 th order

+ 4th order +10 th order

+6 th order +12 th order
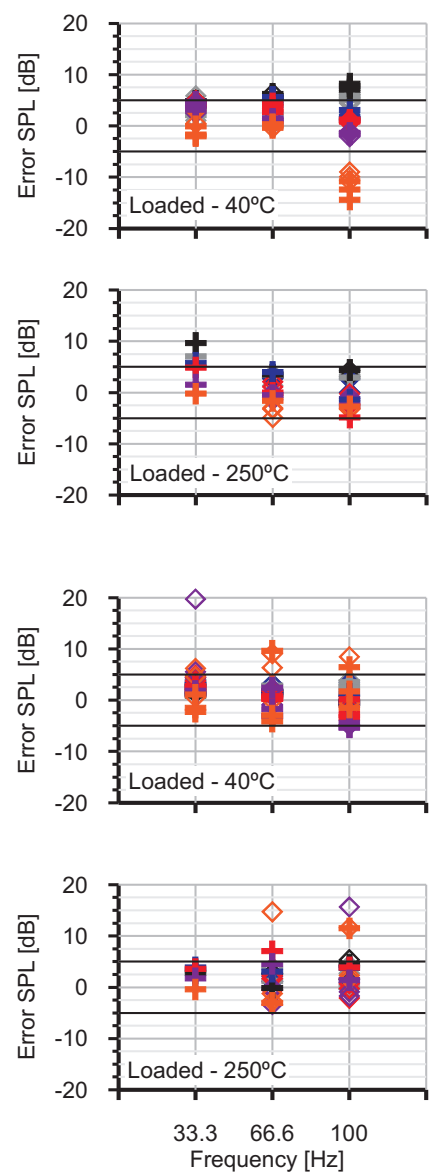

Figure 18: Error in the prediction of reflected and transmitted SPL as a function of pulse amplitude and frequency, mass flow, temperature, DPF soot loading and order of the harmonics. 